\title{
A Diazo-Hooker Reaction, Inspired by the Biosynthesis of Azamerone
}

Oussama Yahiaoui, ${ }^{\text {a }}$ Lauren A. M. Murray, ${ }^{\mathrm{a}}$ Fengyue Zhao, ${ }^{\mathrm{b}}$ Bradley S. Moore,${ }^{\mathrm{c}}$ Kendall N. Houk, ${ }^{\mathrm{d}}$ Fang Liu, ${ }^{*, \mathrm{~b}}$ and Jonathan H. George*,a

a Department of Chemistry, University of Adelaide, Adelaide, SA 5005, Australia.

${ }^{b}$ College of Sciences, Nanjing Agricultural University, Nanjing 210095, China.

${ }^{c}$ Center for Marine Biotechnology and Biomedicine, Scripps Institution of Oceanography, University of California, San Diego, La Jolla, CA 92093, USA.

${ }^{d}$ Department of Chemistry and Biochemistry, University of California, Los Angeles, CA 90095, USA.

\section{Supporting Information}

1. General Methods

2. Experimental Procedures

3. NMR Spectra

4. Selected IR Spectra

5. Computational Studies

\section{S-2}

S-3

S-16

S-35

S-36 


\section{General Methods}

All chemicals were purchased from commercial suppliers and used as received. All reactions were performed under an inert atmosphere of $\mathrm{N}_{2}$. All organic extracts were dried over anhydrous magnesium sulfate or sodium sulfate. Thin layer chromatography was performed using aluminium sheets coated with silica gel $\mathrm{F}_{254}$. Visualization was aided by viewing under a UV lamp and staining with ceric ammonium molybdate or $\mathrm{KMnO}_{4}$ stain followed by heating. All $R_{f}$ values were measured to the nearest 0.05 . Flash column chromatography was performed using 40-63 micron grade silica gel. Melting points were recorded on a digital melting point apparatus and are uncorrected. Infrared spectra were recorded using an FT-IR spectrometer as the neat compounds. NMR spectra were recorded using either a $500 \mathrm{MHz}$ spectrometer $\left({ }^{1} \mathrm{H}\right.$ at $500 \mathrm{MHz},{ }^{13} \mathrm{C}$ at 125 $\mathrm{MHz})$ or $600 \mathrm{MHz}$ spectrometer $\left({ }^{1} \mathrm{H}\right.$ at $600 \mathrm{MHz},{ }^{13} \mathrm{C}$ at $\left.150 \mathrm{MHz}\right) .{ }^{1} \mathrm{H}$ chemical shifts are reported in parts per million (ppm) relative to the tetramethylsilane peak recorded as $\delta 0.00 \mathrm{ppm}$ in $\mathrm{CDCl}_{3} / \mathrm{TMS}$ solvent, or the residual acetone $(\delta 2.05 \mathrm{ppm})$, chloroform $(\delta 7.26 \mathrm{ppm})$, methanol $(\delta 3.31 \mathrm{ppm})$ or DMSO $(\delta 2.50 \mathrm{ppm})$ peaks. The ${ }^{13} \mathrm{C}$ NMR values were referenced to the residual acetone $(\delta 29.9 \mathrm{ppm})$, chloroform $(\delta 77.16 \mathrm{ppm})$, methanol ( $\delta 49.00 \mathrm{ppm}$ ) or DMSO ( $\delta 39.52 \mathrm{ppm}$ ) peaks. Multiplicities are reported as (br) broad, (s) singlet, (d) doublet, (t) triplet, (q) quartet, (quin) quintet, (sext) sextet, (hept) heptet and (m) multiplet. All $J$-values were rounded to the nearest $0.1 \mathrm{~Hz}$. Assignments are made with the aid of COSY, NOESY, HSQC and HMBC experiments. ESI high resolution mass spectra were recorded on an ESI-TOF mass spectrometer. 


\section{Experimental Procedures}

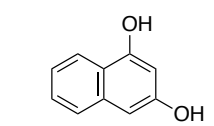

1,3-dihydroxynaphthalene

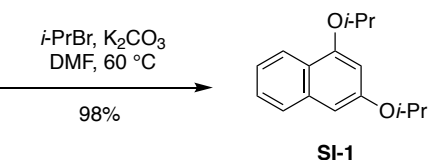

To a solution of 1,3-dihydroxynaphthalene (20.0 g, 0.125 mol, 1.00 eq.) in DMF (400 mL) was added 2bromopropane ( $47.0 \mathrm{ml}, 0.501 \mathrm{~mol}, 4.00$ eq.) and $\mathrm{K}_{2} \mathrm{CO}_{3}(52.0 \mathrm{~g}, 0.376 \mathrm{~mol}, 3.00$ eq.) at room temperature. The reaction was heated at $60^{\circ} \mathrm{C}$ (using an aluminium heating block) for $16 \mathrm{~h}$, then water $(150 \mathrm{~mL})$ was added, and the mixture was extracted with hexane $(150 \mathrm{~mL} \times 3)$, washed with brine $(50 \mathrm{~mL} \times 2)$, dried over $\mathrm{MgSO}_{4}$, filtered, and concentrated in vacuo. The product SI-1 was obtained as a red oil (30.0 g, 98\%). Data for SI-1 matched that previously reported. ${ }^{1}$

Data for SI-1:

$\mathbf{R}_{\mathbf{f}} 0.65$ (hexanes/EtOAc, 4:1)

IR (neat) 2924, 1627, 1597, 1454, 1347, 1373, 1330, $1100 \mathrm{~cm}^{-1}$.

${ }^{1}$ H NMR $\left(500 \mathrm{MHz}, \mathrm{CDCl}_{3}\right) \delta 8.15(1 \mathrm{H}, \mathrm{d}, J=8.4 \mathrm{~Hz}), 7.63(1 \mathrm{H}, \mathrm{d}, J=8.2 \mathrm{~Hz}), 7.40(1 \mathrm{H}, \mathrm{t}, J=7.5 \mathrm{~Hz}), 7.28$ $(1 \mathrm{H}, \mathrm{m}), 6.71(1 \mathrm{H}, \mathrm{s}), 6.49(1 \mathrm{H}, \mathrm{s}), 4.70(2 \mathrm{H}, \mathrm{h}, J=6.0 \mathrm{~Hz}), 1.44(6 \mathrm{H}, \mathrm{d}, J=6.0 \mathrm{~Hz}), 1.41(6 \mathrm{H}, \mathrm{d}, J=6.0 \mathrm{~Hz})$.

${ }^{13} \mathrm{C}$ NMR $\left(125 \mathrm{MHz}, \mathrm{CDCl}_{3}\right) \delta 156.3,155.0,135.5,126.9,126.4,122.7,122.5,122.4,100.8,100.2,70.5,70.4$, $69.9,69.8,22.2$.

${ }^{1}$ K. Grudzien, M. Malinska, M. Barbasiewicz, Organometallics 2012, 31, 3636-3646. 


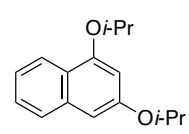

SI-1

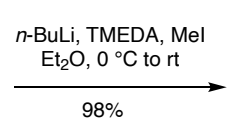

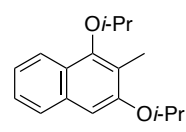

SI-2

To a solution of SI-1 (9.50 g, $38.8 \mathrm{mmol}, 1.00$ eq.) in anhydrous $\mathrm{Et}_{2} \mathrm{O}(50 \mathrm{~mL})$ was added TMEDA (9.0 mL, $58.3 \mathrm{mmol}, 1.50$ eq.) and $n$-BuLi $\left(23.0 \mathrm{~mL}, 2.5 \mathrm{M}\right.$ in hexane, $57.5 \mathrm{mmol}, 1.50$ eq.) at $0{ }^{\circ} \mathrm{C}$. The mixture was stirred for $18 \mathrm{~h}$ at room temperature, then cooled down to $0{ }^{\circ} \mathrm{C}$ and $\mathrm{MeI}(3.6 \mathrm{~mL}, 58.3 \mathrm{mmol}, 1.50$ eq.) was added. The reaction was stirred for $2 \mathrm{~h}$ at room temperature, then quenched with $1 \mathrm{M} \mathrm{HCl}(20 \mathrm{~mL})$ and extracted with hexane $(100 \mathrm{~mL})$. The organic extracts were washed with brine $(50 \mathrm{~mL} \times 2)$, dried over $\mathrm{MgSO}_{4}$, filtered, and concentrated in vacuo. The residue was purified by flash column chromatography on $\mathrm{SiO}_{2}$ (hexanes/EtOAc, 9:1) to afford SI-2 as a yellow oil (9.85 g, 98\%).

Data for SI-2:

$\mathbf{R}_{\mathbf{f}} 0.65$ (hexanes/EtOAc, $4: 1$ )

IR (neat) 2974, 2929, 1625, 1597, 1574, 1498, 1438, 1381, 1324, 1274, 1136, 118, 905, 745, $710 \mathrm{~cm}^{-1}$.

${ }^{1} \mathbf{H}$ NMR $\left(500 \mathrm{MHz}, \mathrm{CDCl}_{3}\right) \delta 8.15(1 \mathrm{H}, \mathrm{d}, J=8.4 \mathrm{~Hz}), 7.63(1 \mathrm{H}, \mathrm{d}, J=8.2 \mathrm{~Hz}), 7.40(1 \mathrm{H}, \mathrm{t}, J=7.5 \mathrm{~Hz}), 7.28$ $(1 \mathrm{H}, \mathrm{m}), 6.71(1 \mathrm{H}, \mathrm{s}), 6.49(1 \mathrm{H}, \mathrm{s}), 4.70(2 \mathrm{H}, \mathrm{h}, J=6.0 \mathrm{~Hz}), 1.44(6 \mathrm{H}, \mathrm{d}, J=6.0 \mathrm{~Hz}), 1.41(6 \mathrm{H}, \mathrm{d}, J=6.0 \mathrm{~Hz})$.

${ }^{13}$ C NMR $\left(125 \mathrm{MHz}, \mathrm{CDCl}_{3}\right) \delta 155.6,152.4,133.4,126.5,125.6,124.8,123.0,122.7,121.4,103.2,76.2,70.2$, 70.1, 22.8, 22.3, 11.1 .

HRMS (ESI-TOF) m/z: [M + H] $]^{+}$Calcd for $\mathrm{C}_{17} \mathrm{H}_{23} \mathrm{O}_{2}$ 259.1693; Found 259.1703. 


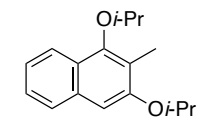

SI-2

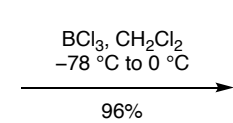

$96 \%$

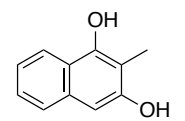

To a solution of SI-2 (0.400 g, $1.55 \mathrm{mmol}, 1.00 \mathrm{eq}$.) in anhydrous $\mathrm{CH}_{2} \mathrm{Cl}_{2}(10 \mathrm{~mL})$ was added $\mathrm{BCl}_{3}(6.19 \mathrm{~mL}$, $1 \mathrm{M}$ in $\mathrm{CH}_{2} \mathrm{Cl}_{2}, 6.19 \mathrm{mmol}, 4.00$ eq.) over $30 \mathrm{~min}$ at $-78{ }^{\circ} \mathrm{C}$. The reaction was stirred at $-78^{\circ} \mathrm{C}$ for $2 \mathrm{~h}$, then 4 h at $0{ }^{\circ} \mathrm{C}$. The mixture was quenched slowly with ice-cold water $(10 \mathrm{~mL})$ at $0{ }^{\circ} \mathrm{C}$ and extracted with EtOAc $(10$ $\mathrm{mL} \times 3)$. The organic extracts were washed with brine $(5 \mathrm{~mL} \times 3)$, dried over $\mathrm{MgSO}_{4}$, filtered, and concentrated in vacuo. The residue was purified by flash column chromatography on $\mathrm{SiO}_{2}$ (hexanes/EtOAc, 9:1 to 4:1 gradient elution) to give 3 as a brown solid (260 mg, 96\%).

Data for 3:

$\mathbf{R}_{\mathbf{f}} 0.30$ (hexanes/EtOAc, $4: 1$ )

MP $115-120^{\circ} \mathrm{C}$

IR (neat) 3400, 2926, 1634, 1590, 1577, 1520, 1386, 1276, 1212, 1180, $1150 \mathrm{~cm}^{-1}$.

${ }^{1}$ H NMR $\left(500 \mathrm{MHz},\left(\mathrm{CD}_{3}\right)_{2} \mathrm{CO}\right) \delta 7.48(1 \mathrm{H}, \mathrm{s}), 7.02-7.00(1 \mathrm{H}, \mathrm{m}), 6.91(1 \mathrm{H}, \mathrm{s}), 6.46-6.45(1 \mathrm{H}, \mathrm{m}), 6.20$ $(1 \mathrm{H}, \mathrm{m}), 6.11(1 \mathrm{H}, \mathrm{m}), 5.75(1 \mathrm{H}, \mathrm{s}), 1.23(1 \mathrm{H}, \mathrm{s})$.

${ }^{13}$ C NMR $\left(125 \mathrm{MHz},\left(\mathrm{CD}_{3}\right) 2 \mathrm{CO}\right) \delta 156.0,151.7,134.4,126.5,126.3,126.3,122.4,121.5,110.7,101.9,9.3$.

HRMS (ESI-TOF) m/z: [M + H] $]^{+}$Calcd for $\mathrm{C}_{11} \mathrm{H}_{11} \mathrm{O}_{2}$ 175.0754; Found 175.0753. 


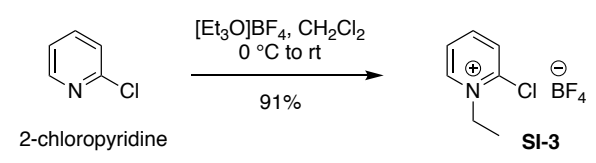

To a solution of 2-chloropyridine (38.0 g, $0.335 \mathrm{~mol}, 1.00$ eq.) in anhydrous $\mathrm{CH}_{2} \mathrm{Cl}_{2}(250 \mathrm{~mL})$ was added triethyloxonium tetrafluoroborate $(64.0 \mathrm{~g}, 0.337 \mathrm{mmol}, 1.00 \mathrm{eq}$. $)$ at $0{ }^{\circ} \mathrm{C}$. The reaction was stirred for $16 \mathrm{~h}$ at room temperature, then the solvent was removed in vacuo to afford SI-3 as a white solid (70.0 g, 91\%). Data for SI-3 matched that previously reported. ${ }^{2}$

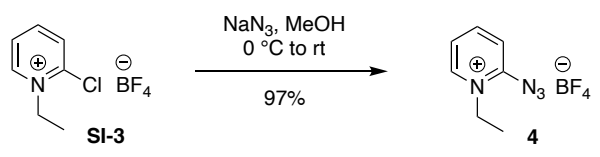

To a solution of SI-3 (15.0 g, 65.0 mmol, 1.00 eq.) in $\mathrm{MeOH}(100 \mathrm{~mL})$ was added $\mathrm{NaN}_{3}(4.25 \mathrm{~g}, 65.4,1.00$ eq.) at $0{ }^{\circ} \mathrm{C}$. The reaction was stirred for $10 \mathrm{~min}$ at room temperature, quenched with $\mathrm{Et}_{2} \mathrm{O}(300 \mathrm{~mL})$ and then cooled at $0{ }^{\circ} \mathrm{C}$ in an ice-bath for $30 \mathrm{~min}$. The resultant precipitate was collected by vacuum filtration to afford the azidinium salt 4 as a white crystalline solid (15.0 g, 97\%). Data for 4 matched that previously reported. ${ }^{3}$

Data for 4:

MP $75-80{ }^{\circ} \mathrm{C}$

IR (neat) 2519, 2143, 1627, 1578, 1504, 14661456, 1320, 1304, 1290, 1172, 975, $791 \mathrm{~cm}^{-1}$.

${ }^{1} \mathbf{H}$ NMR $\left(500 \mathrm{MHz}, \mathrm{D}_{2} \mathrm{O}\right) \delta 8.73(1 \mathrm{H}, \mathrm{d}, J=6.4 \mathrm{~Hz}), 8.61(1 \mathrm{H}, \mathrm{t}, J=6.4 \mathrm{~Hz}), 8.08(1 \mathrm{H}, \mathrm{d}, J=8.5 \mathrm{~Hz}), 7.83$ $(1 \mathrm{H}, \mathrm{t}, J=6.4 \mathrm{~Hz}), 6.67(2 \mathrm{H}, \mathrm{q}, J=7.3 \mathrm{~Hz}), 1.69(3 \mathrm{H}, \mathrm{t}, J=7.3 \mathrm{~Hz})$.

${ }^{13} \mathrm{C}$ NMR $\left(125 \mathrm{MHz}, \mathrm{D}_{2} \mathrm{O}\right) \delta 154.1,149.0,146.1,125.0,121.4,55.3,16.7$.

HRMS (ESI-TOF) m/z: [M] ${ }^{+}$Calcd for $\mathrm{C}_{7} \mathrm{H}_{9} \mathrm{~N}_{4}$ 149.0822; Found 149.0827.

\footnotetext{
${ }^{2}$ M. Kitamura, R. Sakata, N. Tashiro, A. Ikegami, T. Okauchi, Bull. Chem. Soc. Jpn. 2015, 88, 824-833.

${ }^{3}$ H. Balli, F. Kersting, Justus Liebigs Ann. Chem. 1961, 647, 1-10.
} 


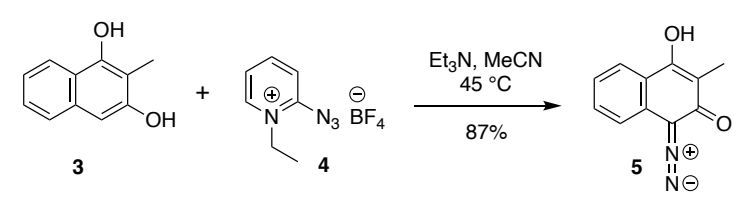

To a solution of 3 ( $1.00 \mathrm{~g}, 5.74 \mathrm{mmol}, 1.00$ eq.) in $\mathrm{MeCN}(100 \mathrm{~mL})$ was added 4 (2.00 g, $8.48 \mathrm{mmol}, 1.50$ eq.) and $\mathrm{Et}_{3} \mathrm{~N}\left(1.60 \mathrm{~mL}, 11.5 \mathrm{mmol}, 2.00\right.$ eq.). The reaction mixture was heated at $45^{\circ} \mathrm{C}$ (using an aluminium heating block) for $3 \mathrm{~h}$, then quenched with a $1 \mathrm{M} \mathrm{HCl}$ solution $(40 \mathrm{~mL})$ and extracted with EtOAc $(50 \mathrm{~mL} \times 3)$. The organic extracts were washed with brine $(20 \mathrm{~mL} \times 3)$, dried over $\mathrm{MgSO}_{4}$, filtered, and concentrated in vacuo. The residue was purified by flash column chromatography on $\mathrm{SiO}_{2}$ (hexanes/EtOAc, 4:1 to 1:1 gradient elution) to afford $\mathbf{5}$ as a brown solid $(1.00 \mathrm{~g}, 87 \%)$.

Data for 5:

$\mathbf{R}_{\mathbf{f}} 0.15$ (hexanes/EtOAc, $3: 2$ )

MP $115-120^{\circ} \mathrm{C}$

IR (neat) 3400, 2956, 2927, 2855, 2100, 1710, 1656, 1645, 1626, 1591, 1458, 1413, 1274, $936 \mathrm{~cm}^{-1}$.

${ }^{1}$ H NMR $\left(500 \mathrm{MHz},\left(\mathrm{CD}_{3}\right)_{2} \mathrm{SO}\right) \delta 7.95(1 \mathrm{H}, \mathrm{d}, J=8.0 \mathrm{~Hz}), 7.46(1 \mathrm{H}, \mathrm{t}, J=7.6 \mathrm{~Hz}), 7.36-7.34(1 \mathrm{H}, \mathrm{m}), 7.25$ $(1 \mathrm{H}, \mathrm{t}, J=7.8 \mathrm{~Hz}), 1.96(3 \mathrm{H}, \mathrm{s})$.

${ }^{13}$ C NMR $\left(125 \mathrm{MHz},\left(\mathrm{CD}_{3}\right)_{2} \mathrm{SO}\right) \delta 179.1,159.3,129.7,126.0,124.5,124.5,122.1,120.4,112.4,73.0,9.3$.

HRMS (ESI-TOF) m/z: $[\mathrm{M}+\mathrm{H}]^{+}$Calcd for $\mathrm{C}_{11} \mathrm{H}_{9} \mathrm{~N}_{2} \mathrm{O}_{2}$ 201.0659; Found 201.0646. 


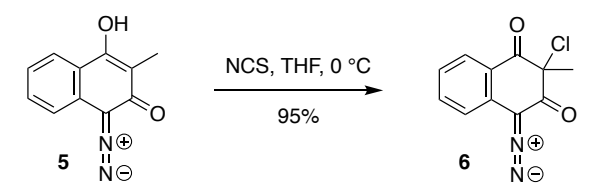

To a solution of 5 (500 mg, $2.50 \mathrm{mmol}, 1.00$ eq.) was added NCS (667 mg, $5.00 \mathrm{mmol}, 2.00$ eq.) at $0{ }^{\circ} \mathrm{C}$. The reaction was stirred for $20 \mathrm{~min}$ at $0{ }^{\circ} \mathrm{C}$, then quenched with brine $(30 \mathrm{~mL})$, extracted with EtOAc $(100 \mathrm{~mL})$, dried over $\mathrm{MgSO}_{4}$, filtered, and concentrated in vacuo. The residue was purified by flash column chromatography on $\mathrm{SiO}_{2}$ (hexanes/EtOAc, 9:1) to afford 6 as a yellow solid (557 mg, 95\%).

Data for 6:

$\mathbf{R}_{\mathbf{f}} 0.65$ (hexanes/EtOAc, $1: 1$ )

MP $112-115^{\circ} \mathrm{C}$

IR (neat): 2097, 1700, 1647, 1600, 1481, 1460, 1359, 1297, $1160 \mathrm{~cm}^{-1}$.

${ }^{1} \mathbf{H}$ NMR $\left(500 \mathrm{MHz}, \mathrm{CDCl}_{3}\right) \delta 8.11(1 \mathrm{H}, \mathrm{d}, J=7.2 \mathrm{~Hz}), 7.66(1 \mathrm{H}, \mathrm{t}, J=7.7 \mathrm{~Hz}), 7.31(1 \mathrm{H}, \mathrm{t}, J=7.6 \mathrm{~Hz}), 7.09$ $(1 \mathrm{H}, \mathrm{d}, J=7.9 \mathrm{~Hz}), 1.93(3 \mathrm{H}, \mathrm{s})$.

${ }^{13}$ C NMR $\left(125 \mathrm{MHz}, \mathrm{CDCl}_{3}\right) \delta 189.2,185.5,135.6,130.1,127.3,126.4,124.9,119.9,65.8,20.0$.

HRMS (ESI-TOF) m/z: [M + H] $]^{+}$Calcd for $\mathrm{C}_{11} \mathrm{H}_{8} \mathrm{ClN}_{2} \mathrm{O}_{2}$ 235.0269; Found 235.0269. 


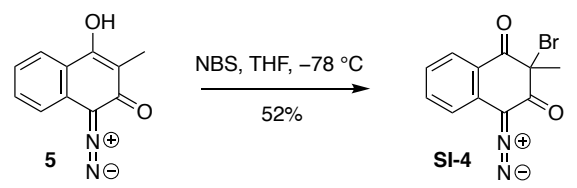

To a solution of 5 (100 mg, $0.500 \mathrm{mmol}, 1.00$ eq.) in THF $(10 \mathrm{~mL})$ at $-78{ }^{\circ} \mathrm{C}$ was added NBS $(133 \mathrm{mg}, 0.747$ mmol, 1.50 eq.). The reaction was stirred at $-78{ }^{\circ} \mathrm{C}$ for $45 \mathrm{~min}$. The reaction mixture was warmed to room temperature, diluted with brine $(20 \mathrm{~mL})$ and extracted with EtOAc $(3 \times 10 \mathrm{~mL})$. The organic extracts were washed with brine $(2 \times 30 \mathrm{~mL})$, dried over anhydrous $\mathrm{MgSO}_{4}$, filtered and concentrated in vacuo. The residue was purified by flash chromatography on $\mathrm{SiO}_{2}$ (hexanes/EtOAc, 5:1) to give $\mathbf{S I - 4}$ as an orange solid (72 mg, $52 \%)$.

Data for SI-4:

$\mathbf{R}_{\mathbf{f}} 0.65$ (hexanes/EtOAc, 1:1)

MP $96-98^{\circ} \mathrm{C}$

IR (neat): 3089, 2544, 2101, 1689, 1641, 1597, 1572, 1482, 1462, 1373, 1355, $1302 \mathrm{~cm}^{-1}$.

${ }^{1} \mathbf{H}$ NMR $\left(500 \mathrm{MHz}, \mathrm{CDCl}_{3}\right): \delta 8.12(1 \mathrm{H}, \mathrm{dd}, J=7.8,1.4 \mathrm{~Hz}), 7.64(1 \mathrm{H}, \mathrm{td}, J=7.7,1.4 \mathrm{~Hz}), 7.31(1 \mathrm{H}, \mathrm{td}, J=$ 7.6, $1.1 \mathrm{~Hz}), 7.09(1 \mathrm{H}, \mathrm{dd}, J=7.9,1.3 \mathrm{~Hz}), 2.07(3 \mathrm{H}, \mathrm{s})$.

${ }^{13}$ C NMR (125 MHz, $\left.\mathrm{CDCl}_{3}\right): \delta 188.9,185.5,135.4,130.0,127.1,126.3,125.0,119.9,71.6,57.0,19.5$. 


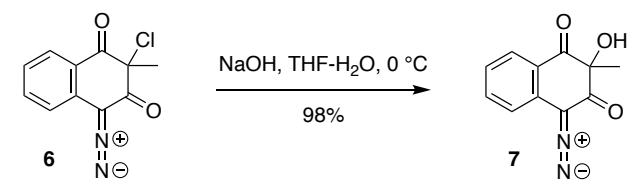

To a solution of $6(50.0 \mathrm{mg}, 0.213 \mathrm{mmol}, 1.00$ eq. $)$ in THF $(3 \mathrm{~mL})$ at $0{ }^{\circ} \mathrm{C}$ was added an ice-cold solution of $\mathrm{NaOH}\left(12.8 \mathrm{mg}, 0.320 \mathrm{mmol}, 1.50\right.$ eq.) in $\mathrm{H}_{2} \mathrm{O}(3 \mathrm{~mL})$. The reaction mixture was stirred for $10 \mathrm{~min}$ at $0{ }^{\circ} \mathrm{C}$, quenched with sat. aqueous $\mathrm{NH}_{4} \mathrm{Cl}(5 \mathrm{~mL})$ and extracted with EtOAc $(10 \mathrm{~mL})$. The organic layers were washed with brine $(5 \mathrm{~mL})$, dried over $\mathrm{MgSO}_{4}$, filtered, and concentrated in vacuo. The residue was purified by flash column chromatography on $\mathrm{SiO}_{2}$ (hexanes/EtOAc, 4:1) to afford 7 as a yellow solid (45 mg, $98 \%$ ).

Data for 7:

$\mathbf{R}_{\mathbf{f}} 0.15$ (hexanes/EtOAc, 3:2)

MP $105-110^{\circ} \mathrm{C}$

IR (neat) 2096, 1698, 1651, 1598, 1570, 1460, 1444, 1300, 1209, 1172, 1108, 1099, 1023, $959 \mathrm{~cm}^{-1}$.

${ }^{1} \mathbf{H}$ NMR $\left(500 \mathrm{MHz}, \mathrm{CDCl}_{3}\right) \delta 8.01(1 \mathrm{H}, \mathrm{d}, J=7.8 \mathrm{~Hz}), 7.64(\mathrm{t}, J=7.2 \mathrm{~Hz}), 7.28(1 \mathrm{H}, \mathrm{t}, J=7.7 \mathrm{~Hz}), 7.09(1 \mathrm{H}$, $\mathrm{d}, J=7.2 \mathrm{~Hz}), 1.57(3 \mathrm{H}, \mathrm{s})$.

${ }^{13}$ C NMR (125 MHz, $\left.\mathrm{CDCl}_{3}\right)$ 196.4, 190.8, 135.6, 129.2, 128.1, 126.2, 125, 120.2, 82.8, 28.2, 28.2.

HRMS (ESI-TOF) m/z: [M + H] $]^{+}$Calcd for $\mathrm{C}_{11} \mathrm{H}_{9} \mathrm{~N}_{2} \mathrm{O}_{3}$ 216.0535; Found 216.0542. 


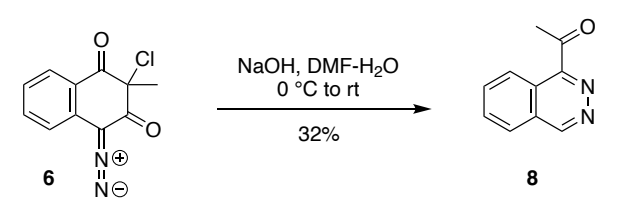

To a solution of 16 (30.0 mg, $0.128 \mathrm{mmol}, 1.00 \mathrm{eq}$.) in DMF (3 mL) was added an ice-cold solution of LiOH (9.2 mg, $0.38 \mathrm{mmol}, 3.0$ eq.) in $\mathrm{H}_{2} \mathrm{O}(3 \mathrm{~mL})$ at $0{ }^{\circ} \mathrm{C}$. The reaction mixture was stirred for $20 \mathrm{~min}$ at $0{ }^{\circ} \mathrm{C}$ and then for $1 \mathrm{~h}$ at room temperature. The mixture was quenched with sat. aqueous $\mathrm{NH}_{4} \mathrm{Cl}(3 \mathrm{~mL})$ and extracted with EtOAc $(10 \mathrm{~mL})$. The organic layers were washed with $0.5 \mathrm{M}$ aqueous $\mathrm{NaOH}(3 \times 5 \mathrm{~mL})$, brine $(5 \mathrm{~mL})$, dried over $\mathrm{MgSO}_{4}$, filtered, and concentrated in vacuo. The residue was purified by flash column chromatography on $\mathrm{SiO}_{2}$ (hexanes/EtOAc, 7:1) to afford 8 as a yellow solid (7 mg, $32 \%$ ). Data for 8 matched that previously reported. ${ }^{4}$

MP $76-78^{\circ} \mathrm{C}$

$\mathbf{R}_{\mathbf{f}} 0.30$ (hexanes/EtOAc, $1: 1$ )

IR (neat): $1740,1350,1200,955,950,750 \mathrm{~cm}^{-1}$.

${ }^{1} \mathbf{H}$ NMR (500 MHz, CDCl $): 9.61(1 \mathrm{H}, \mathrm{s}), 8.98(1 \mathrm{H}, \mathrm{d}, J=8.1 \mathrm{~Hz}), 8.03-7.94(3 \mathrm{H}, \mathrm{m}), 2.98(3 \mathrm{H}, \mathrm{s})$.

${ }^{13}$ C NMR (125 MHz, $\left.\mathrm{CDCl}_{3}\right)$ : 201.7, 153.5, 153.4, 152.5, 134.4, 132.8, 127.6, 126.9, 126.2, 126.2, 124.3, 28.8.

HRMS (ESI-TOF) m/z: [M + H] $]^{+}$Calcd for $\mathrm{C}_{10} \mathrm{H}_{9} \mathrm{~N}_{2} \mathrm{O}$ 173.0709; Found 173.0739.

${ }^{4}$ X. Wang, C. Zeng, Tetrahedron 2019, 75, 1425-1430. 

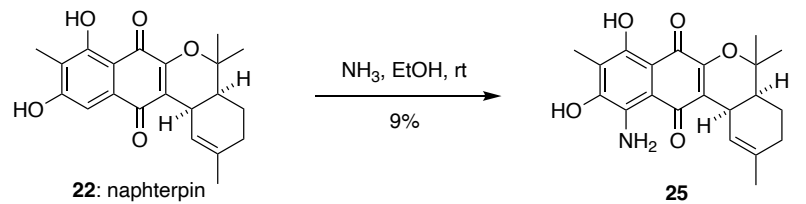

To a solution of naphterpin (22) (20 mg, $0.056 \mathrm{mmol}, 1.00$ eq.) in EtOH (2.5 mL) was added a solution of $\mathrm{NH}_{3}$ ( $30 \%$ in $\mathrm{H}_{2} \mathrm{O}, 16.5 \mathrm{~mL}, 291 \mathrm{mmol}$ ) at room temperature, and the reaction was stirred for $36 \mathrm{~h}$. The mixture was then cooled to $0{ }^{\circ} \mathrm{C}$ and acidified with $10 \%$ aqueous $\mathrm{H}_{2} \mathrm{SO}_{4}(20 \mathrm{~mL})$ to $\mathrm{pH} 1$. The mixture was extracted with EtOAc $(3 \times 10 \mathrm{~mL})$. The combined organic extracts were washed sequentially with saturated aqueous $\mathrm{NaHCO}_{3}$ solution $(30 \mathrm{~mL})$ and brine $(30 \mathrm{~mL})$, dried over $\mathrm{MgSO}_{4}$, filtered and concentrated in vacuo. The residue was purified by flash chromatography on $\mathrm{SiO}_{2}$ (hexanes/EtOAc, $2: 1$ to 3:7, gradient elution) to give $\mathbf{2 5}$ ( $2 \mathrm{mg}, 9 \%$ ) as a magenta residue.

Data for 25:

$\mathbf{R}_{\mathbf{f}} 0.20$ (hexanes/EtOAc, $1: 1$ )

IR (neat) 3488, 3294, 3143, 2928, 1595, 1564, 1510, 1467, 1439, 1339, $1276 \mathrm{~cm}^{-1}$.

${ }^{1}$ H NMR $\left(600 \mathrm{MHz}, \mathrm{CDCl}_{3}\right): \delta 14.17(\mathrm{~s}, 1 \mathrm{H}), 6.20(\mathrm{~d}, J=5.1 \mathrm{~Hz}, 1 \mathrm{H}), 3.55-3.51(\mathrm{~m}, 1 \mathrm{H}), 2.18(\mathrm{~s}, 3 \mathrm{H}), 2.04$ $-1.90(\mathrm{~m}, 3 \mathrm{H}), 1.77(\mathrm{ddd}, J=11.8,6.2,2.8 \mathrm{~Hz}, 1 \mathrm{H}), 1.68(\mathrm{~s}, 3 \mathrm{H}), 1.54(\mathrm{~s}, 3 \mathrm{H}), 1.39-1.35(\mathrm{~m}, 1 \mathrm{H}), 1.33$ (s, $3 \mathrm{H})$.

${ }^{13}$ C NMR (150 MHz, $\left.\mathrm{CDCl}_{3}\right): \delta 184.9,181.2,159.6,152.2,150.9,137.1,135.7,125.4,121.1,114.8,106.9$, $106.5,79.7,40.2,31.8,29.8,25.8,25.0,23.8,20.8,8.0$.

HRMS (ESI-TOF) m/z: [M + H] Calcd for $\mathrm{C}_{21} \mathrm{H}_{24} \mathrm{NO}_{5}$ 370.1649; Found 370.1671. 

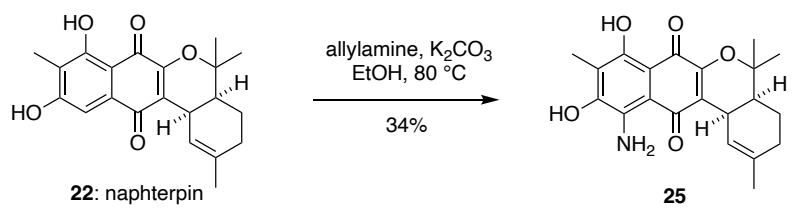

To a solution of naphterpin (22) $(207 \mathrm{mg}, 0.584 \mathrm{mmol}, 1.00$ eq.) in EtOH (10 mL) at room temperature was added $\mathrm{K}_{2} \mathrm{CO}_{3}$ (805 mg, $5.83 \mathrm{mmol}, 10.0$ eq.) and allylamine (5.00 mL, $67.2 \mathrm{mmol}, 115$ eq.). The reaction was heated at $80^{\circ} \mathrm{C}$ (using an aluminium heating block) for $16 \mathrm{~h}$. The reaction mixture was quenched with $1 \mathrm{M} \mathrm{HCl}$ $(50 \mathrm{~mL})$ and extracted with EtOAc $(3 \times 20 \mathrm{~mL})$. The combined organic extracts were washed with brine $(100$ $\mathrm{mL}$ ), dried over $\mathrm{Na}_{2} \mathrm{SO}_{4}$, filtered and concentrated in vacuo. The residue was purified by flash chromatography on $\mathrm{SiO}_{2}$ (hexanes/EtOAc, 4:1 to 1:1, gradient elution) to give $\mathbf{2 5}(81 \mathrm{mg}, 34 \%)$ as a purple residue. Data for $\mathbf{2 5}$ matched that previously obtained. 

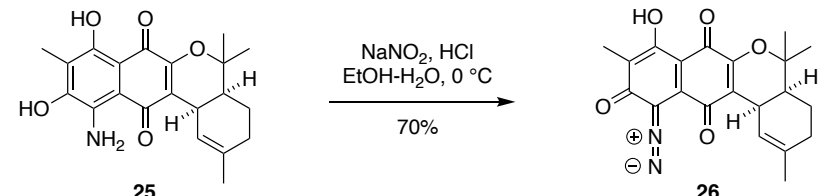

To a solution of 25 (43.0 mg, $0.116 \mathrm{mmol}, 1.00$ eq. $)$ in $\mathrm{EtOH}(2 \mathrm{~mL})$ at $0{ }^{\circ} \mathrm{C}$ was added $32 \%$ aqueous $\mathrm{HCl}(0.10$ $\mathrm{mL}, 1.05 \mathrm{mmol}, 9.05 \mathrm{eq})$. The reaction was stirred at $0{ }^{\circ} \mathrm{C}$ for $5 \mathrm{~min}$. A solution of $\mathrm{NaNO}_{2}(22.0 \mathrm{mg}, 0.318$ mmol, 2.74 eq.) in $\mathrm{H}_{2} \mathrm{O}(0.5 \mathrm{~mL})$ at $0{ }^{\circ} \mathrm{C}$ was then added dropwise. The resulting mixture was stirred at $0{ }^{\circ} \mathrm{C}$ for $30 \mathrm{~min}$. The mixture was diluted with $\mathrm{CH}_{2} \mathrm{Cl}_{2}(10 \mathrm{~mL})$, and a solution of $\mathrm{K}_{2} \mathrm{CO}_{3}(97 \mathrm{mg}, 0.70 \mathrm{mmol}, 6.00 \mathrm{eq}$.) in $\mathrm{H}_{2} \mathrm{O}(1 \mathrm{~mL})$ was added at $0{ }^{\circ} \mathrm{C}$. The reaction was stirred vigorously at $0{ }^{\circ} \mathrm{C}$ for $2 \mathrm{~min}$. The organic phase was then separated, and the aqueous phase extracted with $\mathrm{CH}_{2} \mathrm{Cl}_{2}(2 \times 10 \mathrm{~mL})$. The combined organic extracts were washed with brine $(30 \mathrm{~mL})$, dried over $\mathrm{Na}_{2} \mathrm{SO}_{4}$, filtered and concentrated in vacuo (at $25^{\circ} \mathrm{C}$ ). The residue was purified by flash chromatography on $\mathrm{SiO}_{2}$ (hexanes/EtOAc, 2:1) to give 26 (31 $\mathrm{mg}, 70 \%$ ) as a dark green residue.

Data for 26:

$\mathbf{R}_{\mathbf{f}} 0.55$ (hexanes/EtOAc, $\left.1: 1\right)$

IR (neat) 2928, 2152, 1642, 1583, 1521, 1431, 1389, 1368, $1333 \mathrm{~cm}^{-1}$.

${ }^{1}$ H NMR (600 MHz, CDCl $) \delta 11.34(\mathrm{~s}, 1 \mathrm{H}), 6.00(\mathrm{~d}, J=5.1 \mathrm{~Hz}, 1 \mathrm{H}), 3.45-3.41(\mathrm{~m}, 1 \mathrm{H}), 2.07(\mathrm{~s}, 3 \mathrm{H}), 2.04$ - $1.92(\mathrm{~m}, 3 \mathrm{H}), 1.26(\mathrm{ddd}, J=12.4,6.1,3.0 \mathrm{~Hz}, 1 \mathrm{H}), 1.69$ (s, 3H), 1.56 (s, 3H), $1.34(\mathrm{~s}, 3 \mathrm{H}), 1.31-1.21(\mathrm{~m}$, $1 \mathrm{H})$.

${ }^{13}$ C NMR (150 MHz, $\left.\mathbf{C D C l}_{3}\right) \delta 181.7,180.9,174.6,161.0,152.7,137.0,133.6,121.2,119.4,119.3,109.4$, $81.9,39.7,31.0,29.8,25.7,25.3,23.7,20.4,8.7$.

${ }^{1}$ H NMR (600 MHz, C $\left.6 \mathbf{D}_{6}\right) \delta 11.59(\mathrm{~s}, 1 \mathrm{H}), 6.23-6.19(\mathrm{~m}, 1 \mathrm{H}), 3.13-3.10(\mathrm{~m}, 1 \mathrm{H}), 2.39(\mathrm{~s}, 3 \mathrm{H}), 1.68(\mathrm{~d}, J$ $=6.6 \mathrm{~Hz}, 2 \mathrm{H}), 1.61(\mathrm{~s}, 3 \mathrm{H}), 1.53-1.48(\mathrm{~m}, 1 \mathrm{H}), 1.13(\mathrm{~s}, 3 \mathrm{H}), 1.11-1.04(\mathrm{~m}, 2 \mathrm{H}), 0.88(\mathrm{~s}, 3 \mathrm{H})$.

${ }^{13}$ C NMR (150 MHz, $\left.\mathbf{C}_{6} \mathbf{D}_{6}\right) \delta 181.4,180.5,174.0,160.7,152.6,135.9,133.5,128.6,120.4,120.4,118.5$, $109.5,80.8,39.3,31.0,29.7,25.3,24.7,23.6,20.4,8.9$.

HRMS (ESI-TOF) m/z: [M + H] $]^{+}$Calcd for $\mathrm{C}_{21} \mathrm{H}_{21} \mathrm{~N}_{2} \mathrm{O}_{5}$ 381.1445; Found 381.1449. 

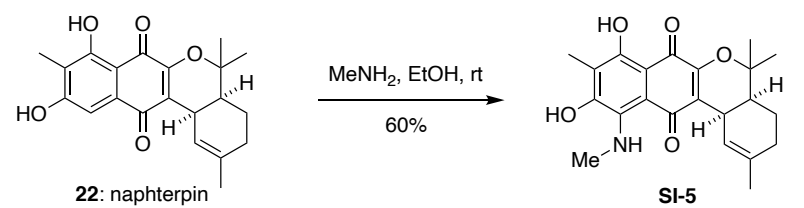

To a solution of naphterpin (22) (50 mg, 0.14 mmol, 1.00 eq.) in $\mathrm{EtOH}(5 \mathrm{~mL})$ was added $\mathrm{MeNH}_{2}(40 \mathrm{~mL}, 33 \%$ in $\mathrm{EtOH}$ ) at room temperature and the reaction was stirred for $16 \mathrm{~h}$. The mixture was acidified with $10 \% \mathrm{H}_{2} \mathrm{SO}_{4}$ to $\mathrm{pH} 1$ and extracted with EtOAc $(3 \times 15 \mathrm{~mL})$. The combined organic extracts were washed sequentially with saturated aqueous $\mathrm{NaHCO}_{3}$ solution $(40 \mathrm{~mL})$ and brine $(40 \mathrm{~mL})$, dried over $\mathrm{MgSO}_{4}$, filtered and concentrated in vacuo. The residue was purified by flash chromatography on $\mathrm{SiO}_{2}$ (hexanes/EtOAc, 2:1) to give SI-5 (32 mg, $60 \%$ ) as a purple residue.

Data for SI-5:

$\mathbf{R}_{\mathbf{f}} 0.10(2: 1$, petrol/EtOAc $)$

IR (neat) 2928, 2906, 1559, 1502, 1424, 1378, $1339 \mathrm{~cm}^{-1}$.

${ }^{1} \mathrm{H}$ NMR (500 MHz, CDCl $) \delta 13.70(\mathrm{~s}, 1 \mathrm{H}), 6.08$ (d, $\left.J=4.6 \mathrm{~Hz}, 1 \mathrm{H}\right), 3.49$ (s, 1H), 2.85 (s, 3H), 2.17 (s, 3H), $2.03-1.91(\mathrm{~m}, 3 \mathrm{H}), 1.77$ (ddd, $J=11.8,6.3,2.8 \mathrm{~Hz}, 1 \mathrm{H}), 1.68(\mathrm{~s}, 3 \mathrm{H}), 1.54(\mathrm{~s}, 3 \mathrm{H}), 1.33(\mathrm{~s}, 3 \mathrm{H}), 1.37-1.29$ $(\mathrm{m}, 1 \mathrm{H})$.

${ }^{13}$ C NMR (125 MHz, $\left.\mathbf{C D C l}_{3}\right) \delta 185.8,181.6,161.7,157.0,152.3,137.3,136.0,124.9,120.6,116.7,116.4$, $106.9,80.1,40.1,35.3,31.5,29.8,25.8,25.1,23.8,20.7,8.2$.

HRMS (ESI-TOF) m/z: [M + H] $]^{+}$Calcd for $\mathrm{C}_{22} \mathrm{H}_{26} \mathrm{NO}_{5}$ 384.1805; Found 384.1823. 


\section{NMR Spectra}

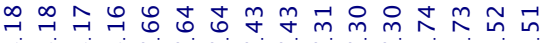

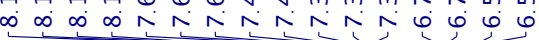

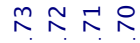

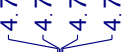

ร ํำ ๆ F

西然i

${ }^{1} \mathrm{HNR}$ spectrum
$\mathrm{CDCl}_{3}$
$500 \mathrm{MHz}$

SI-1
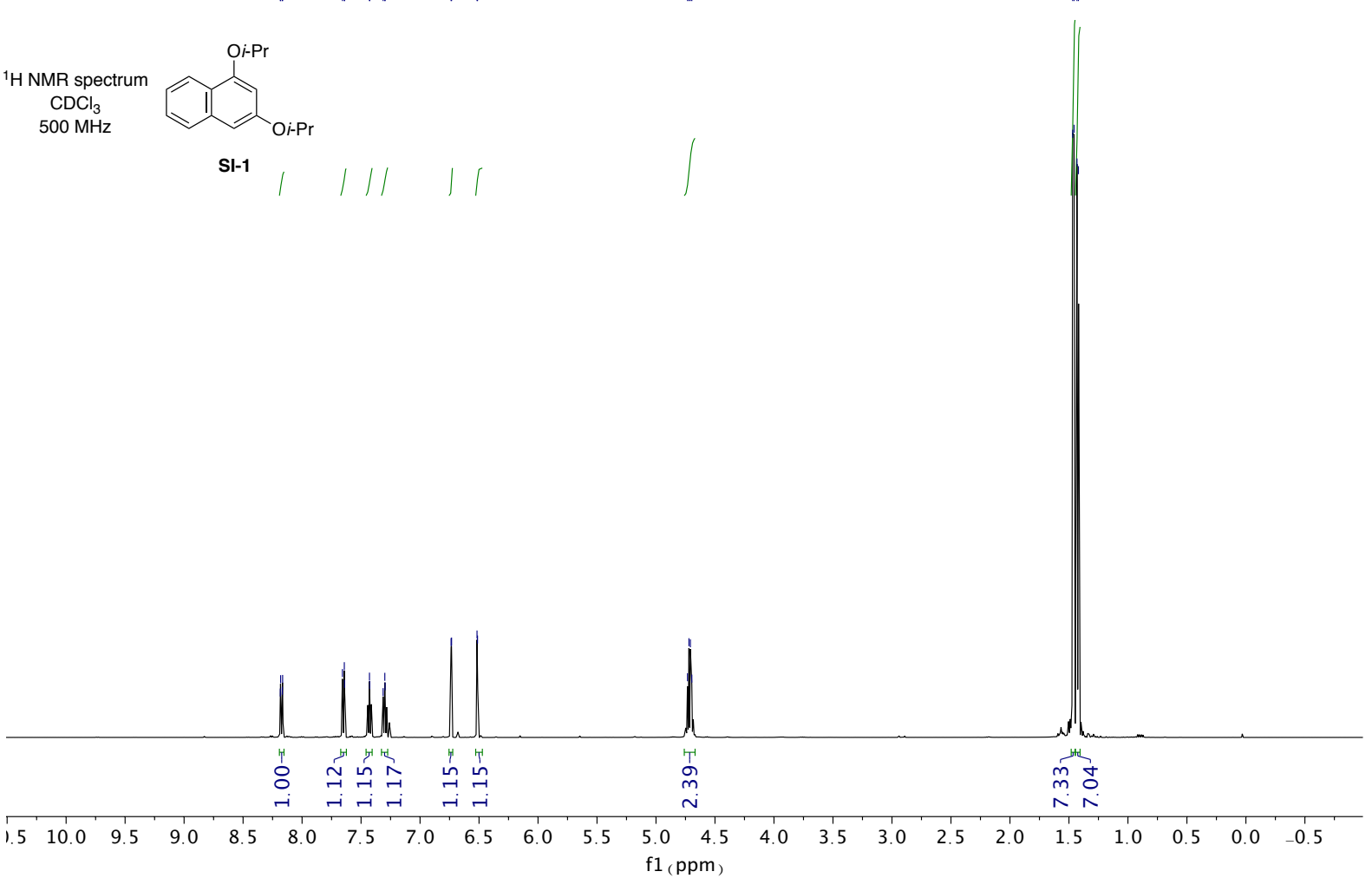

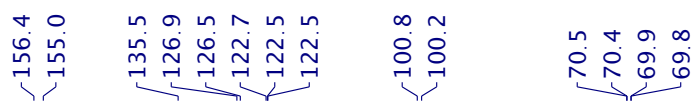

$\stackrel{\sim}{\sim}$

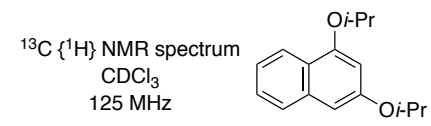

SI-1

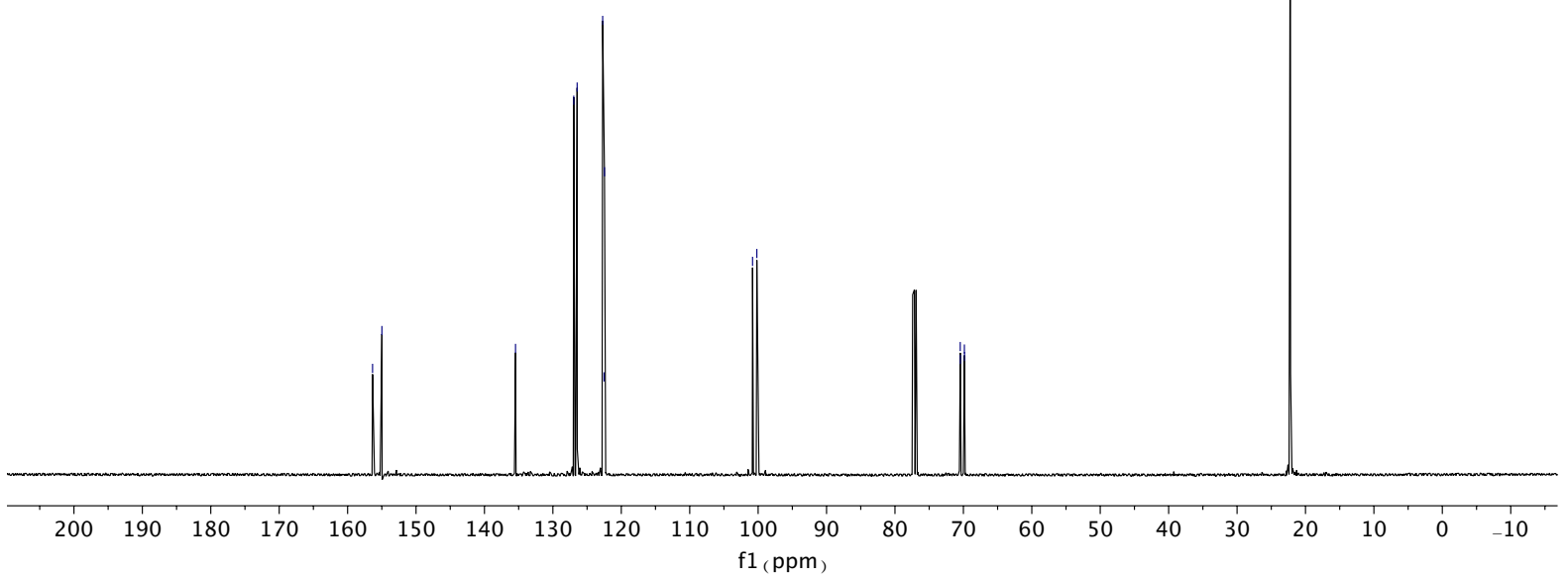




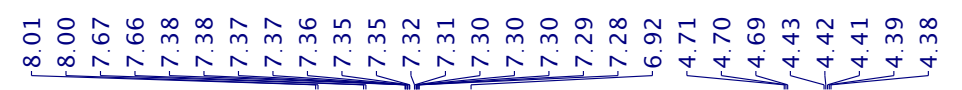
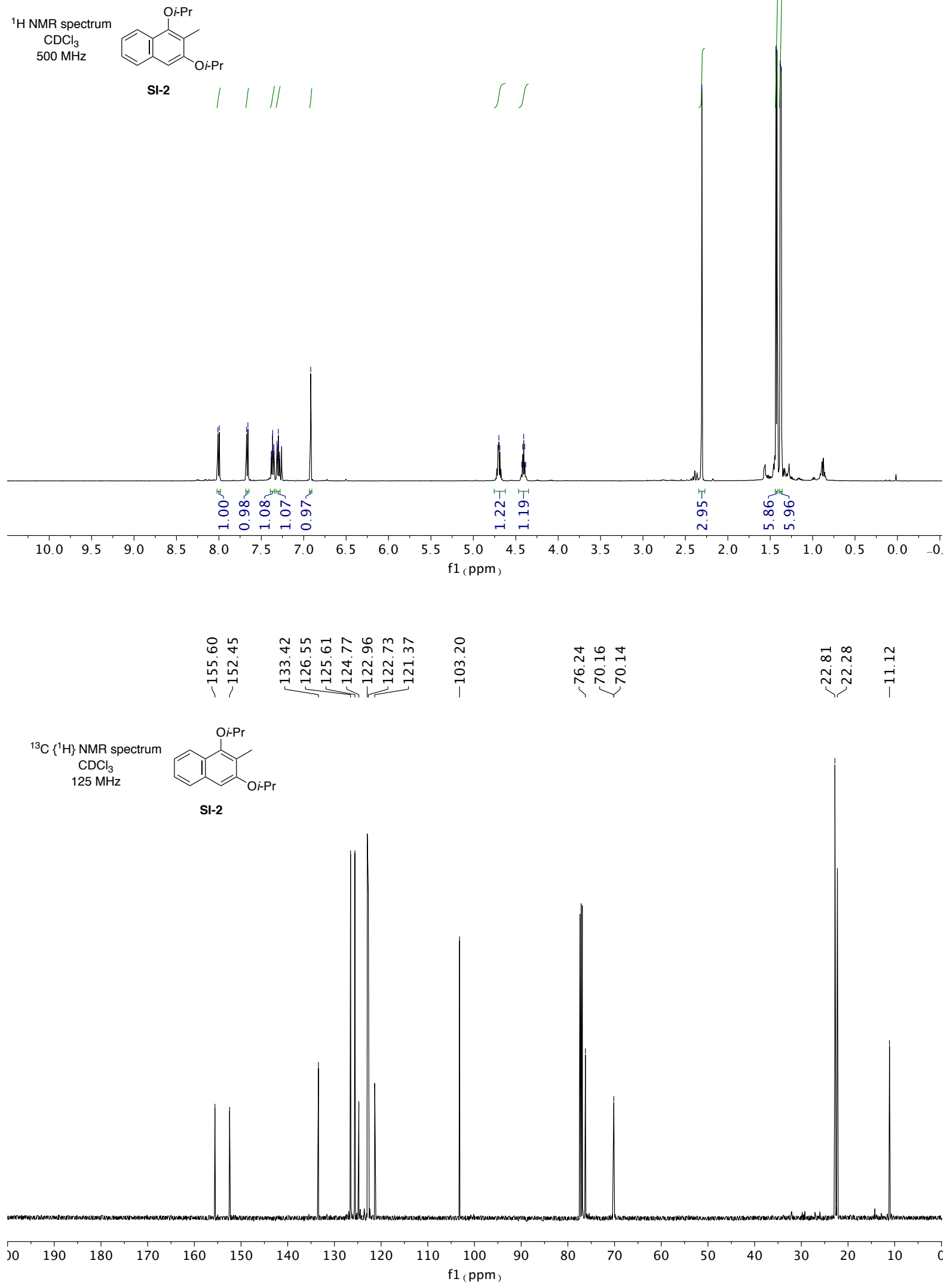

S-17 


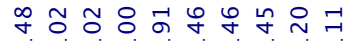

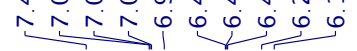

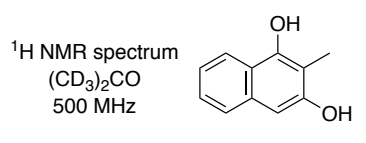

3
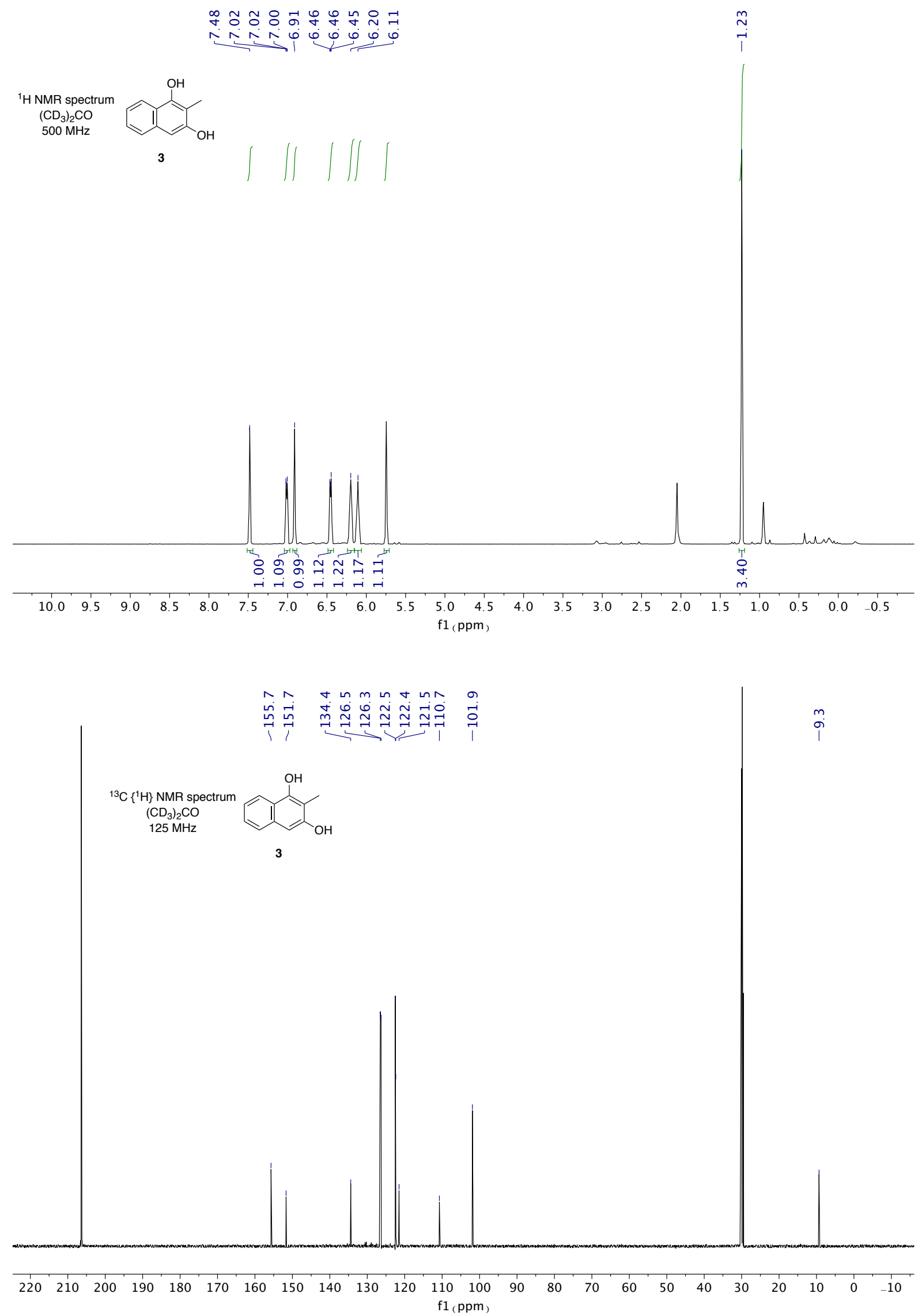


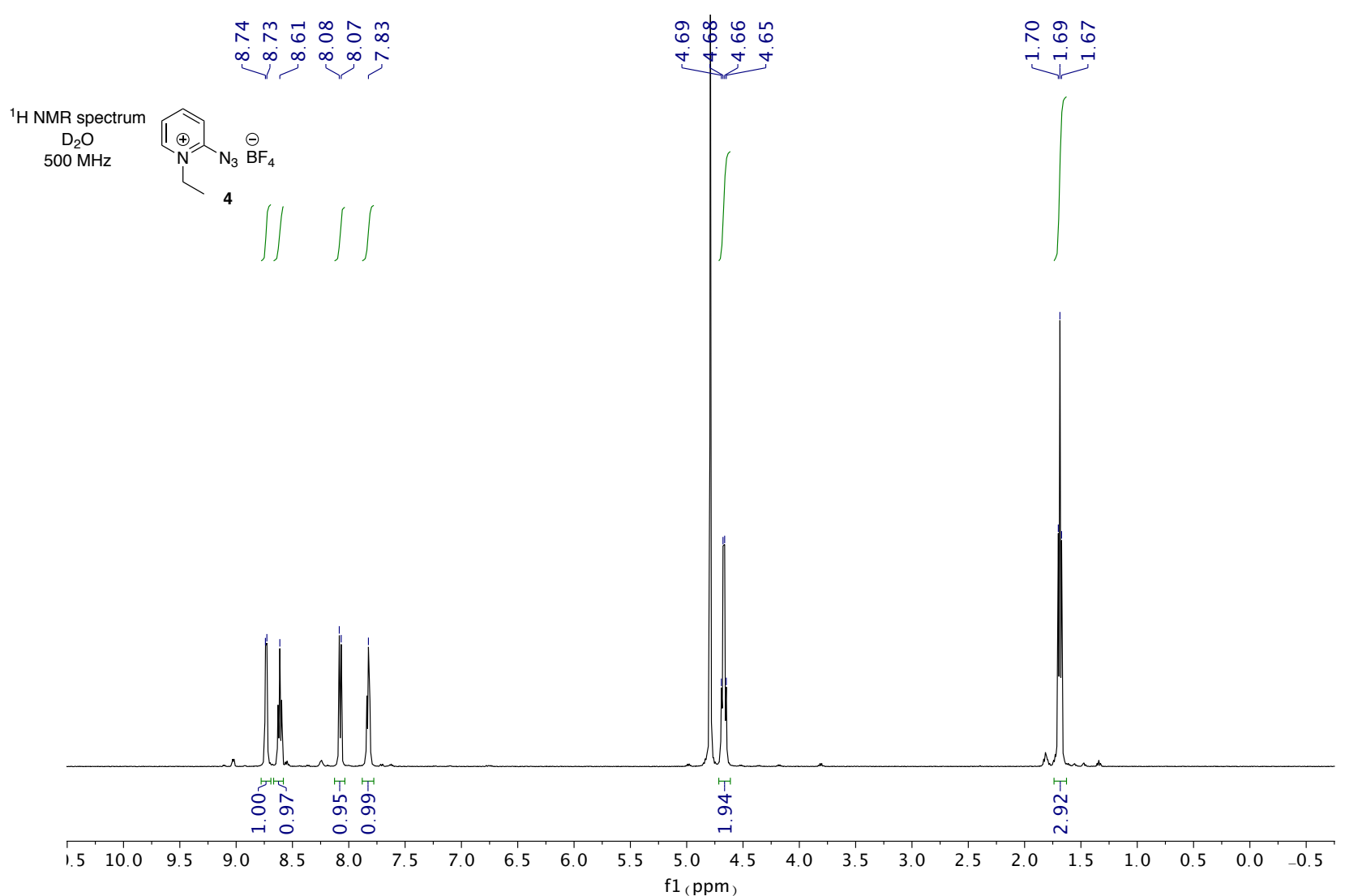

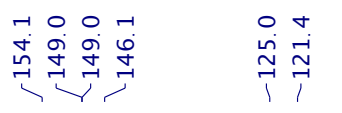

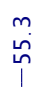

$\hat{0}$

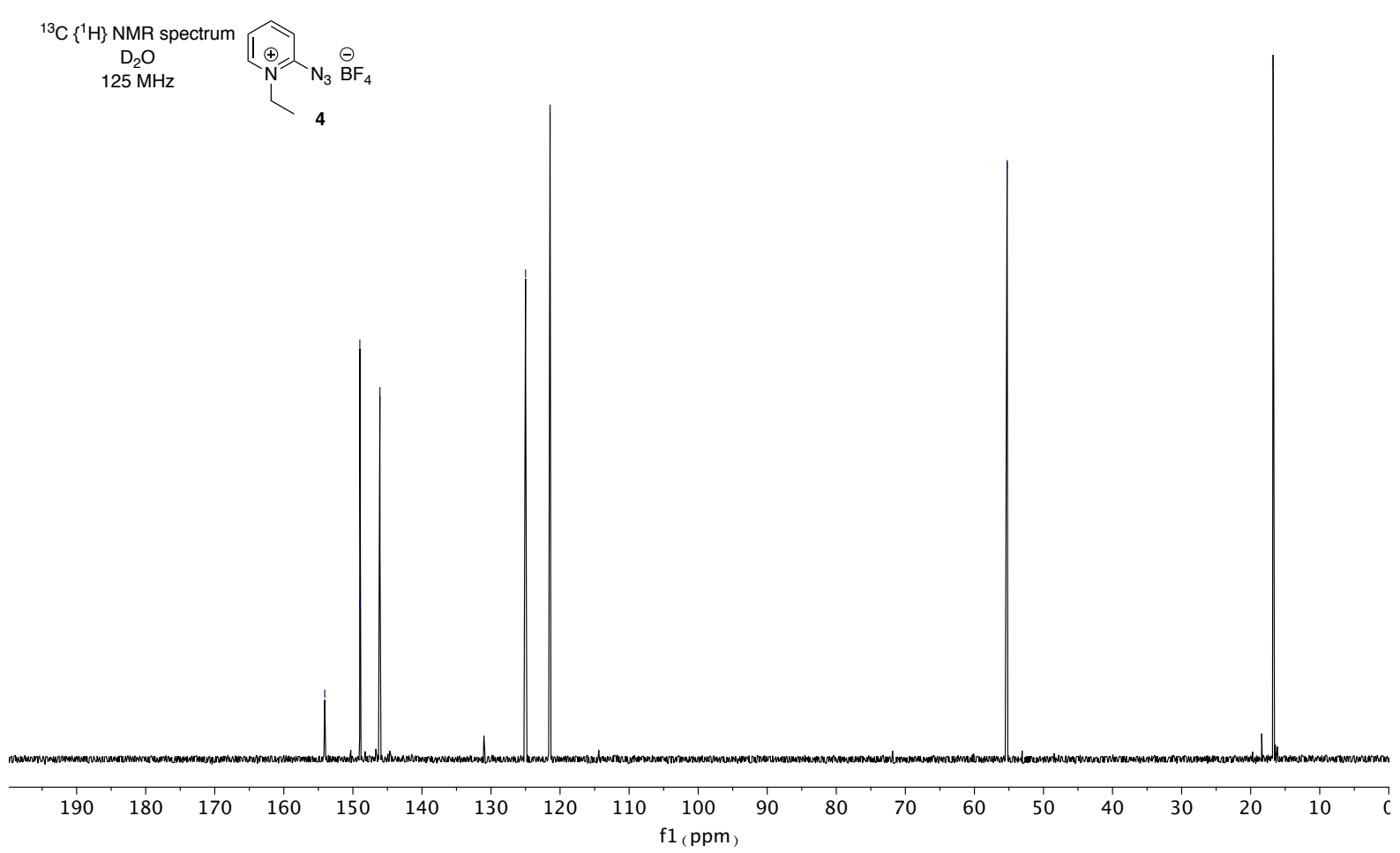

S-19 


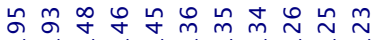

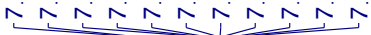
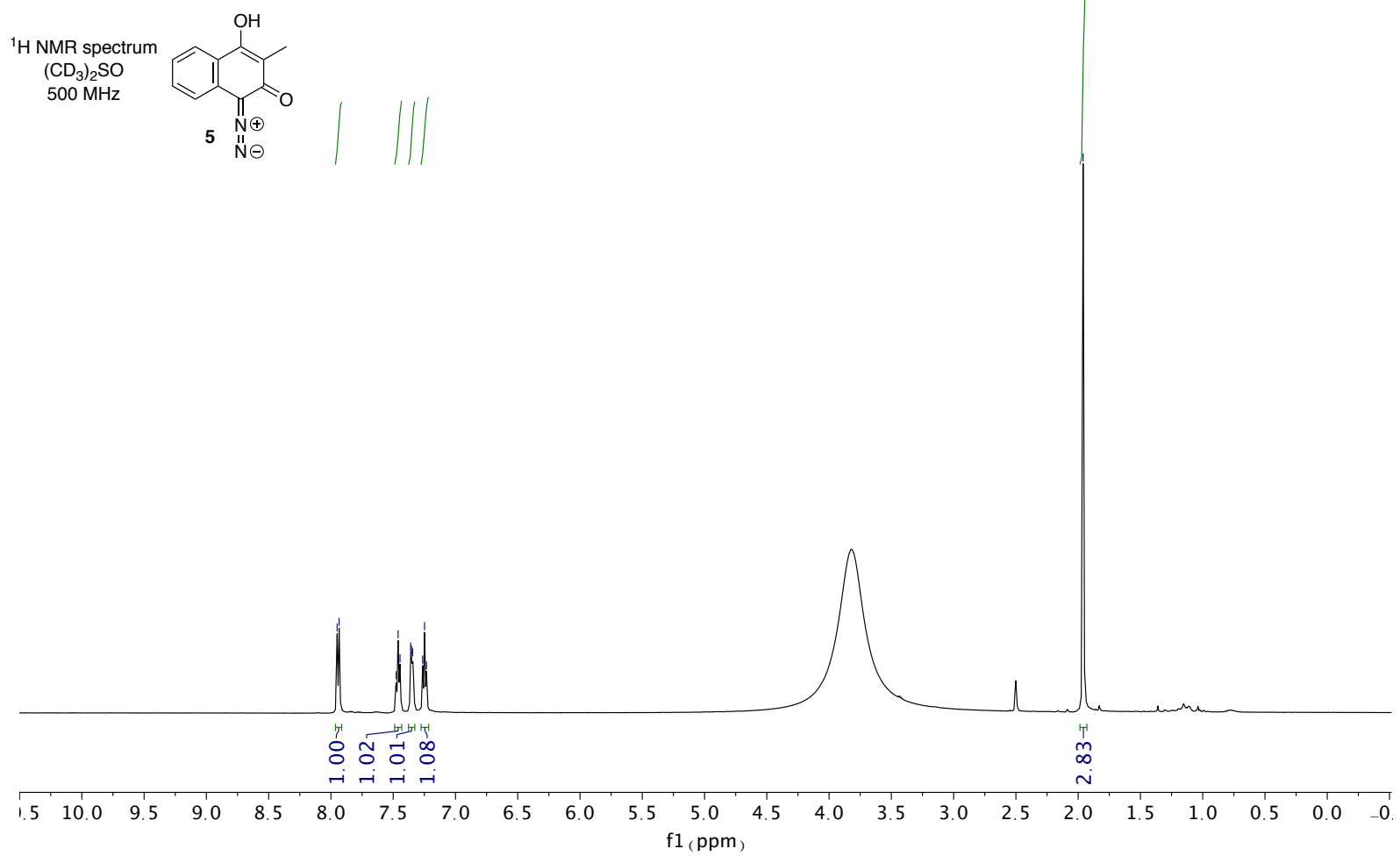

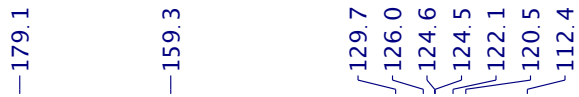

$\stackrel{\circ}{i}$

mi

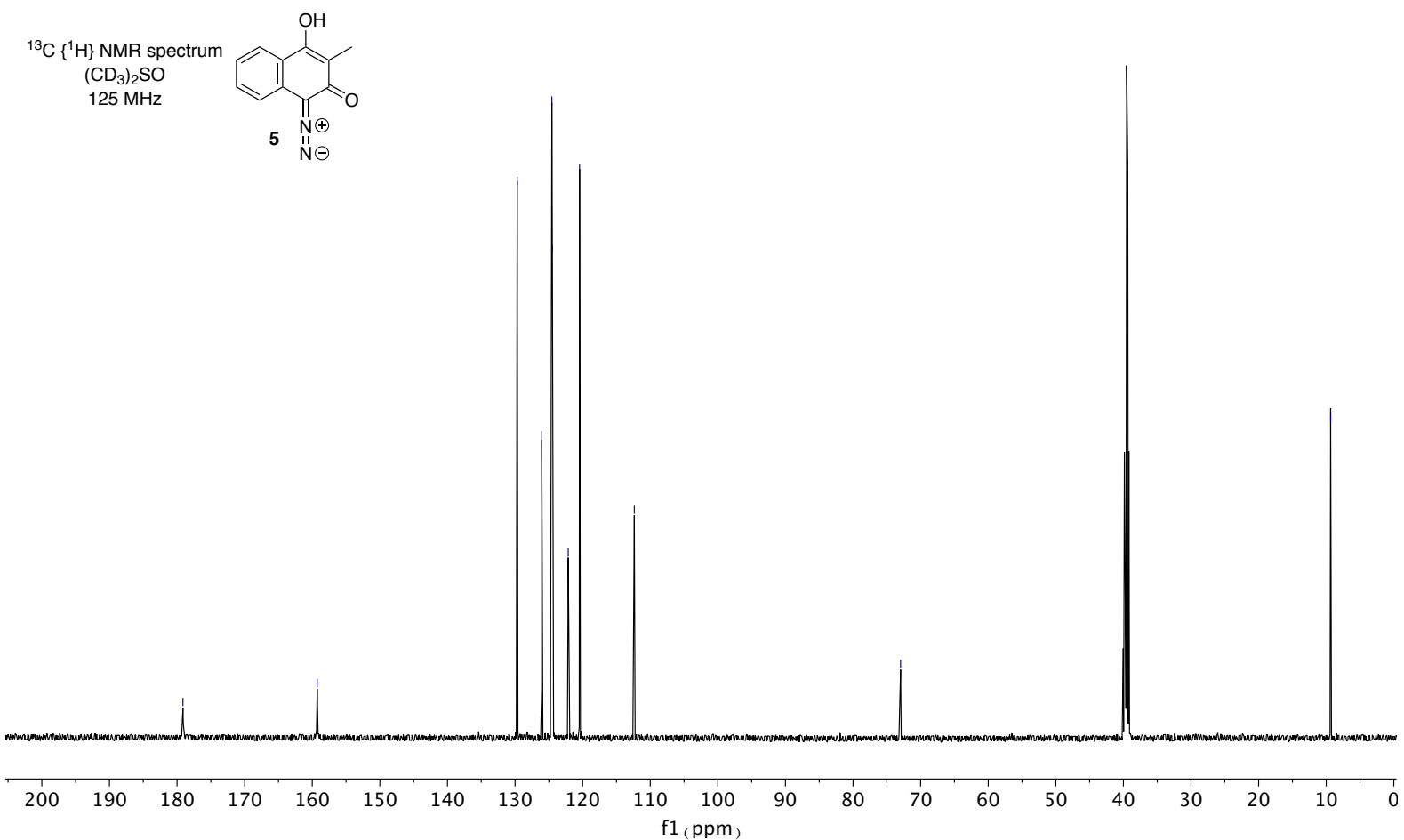




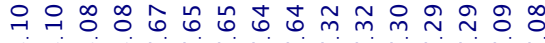

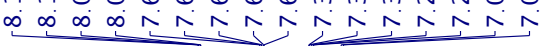
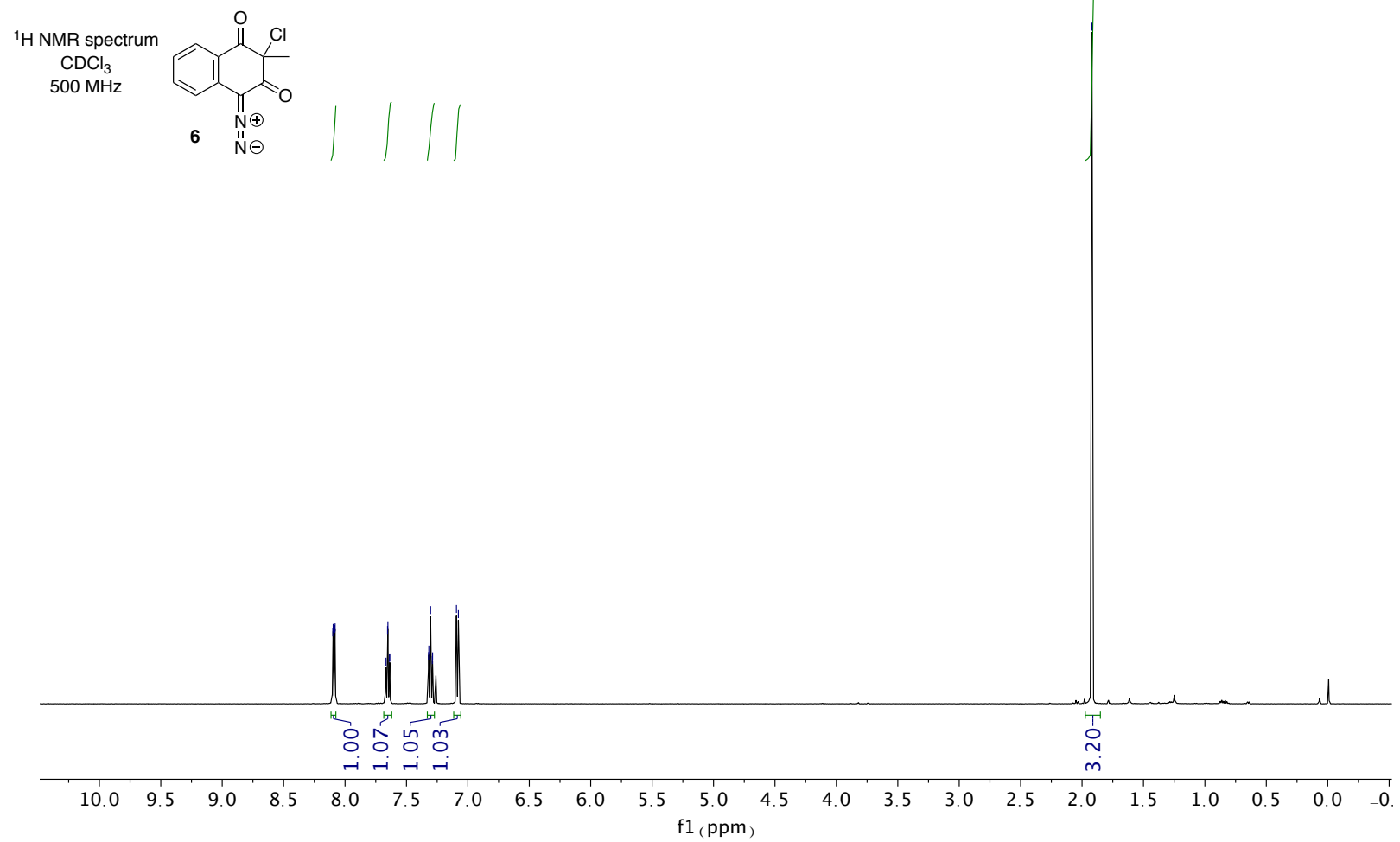

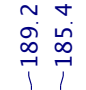

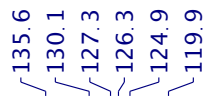

ڤి

$\because 0$

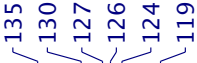

ㅇํํ 요
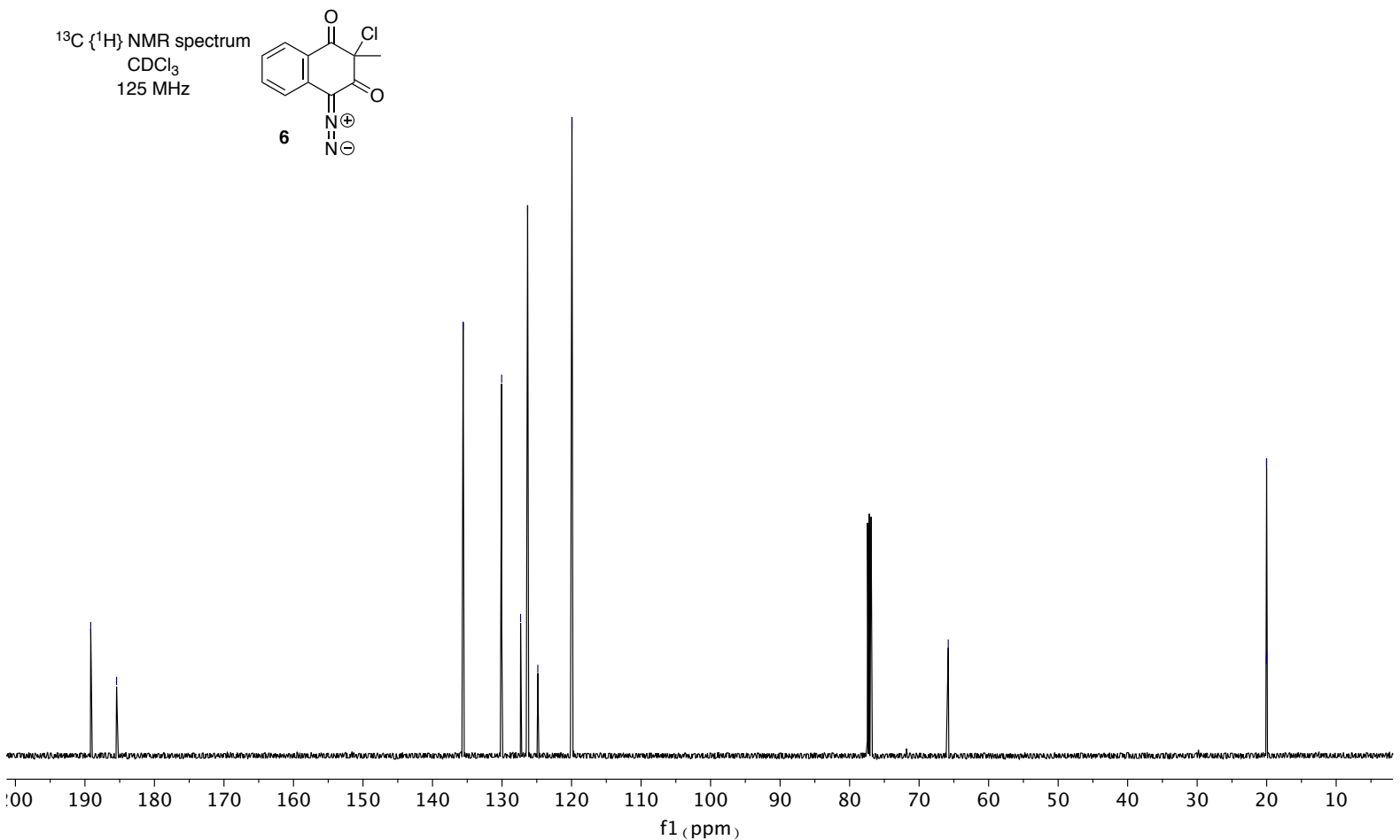

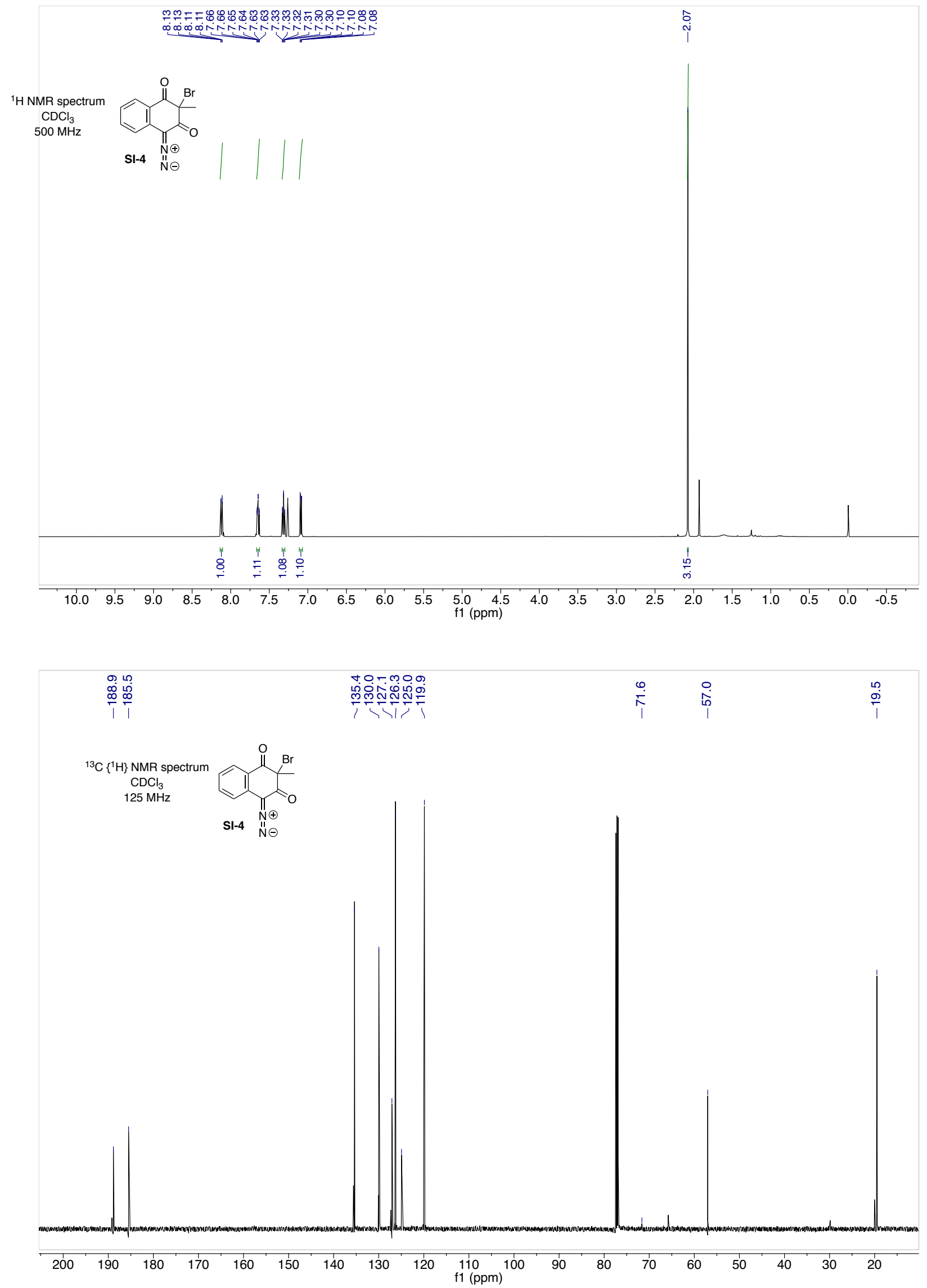

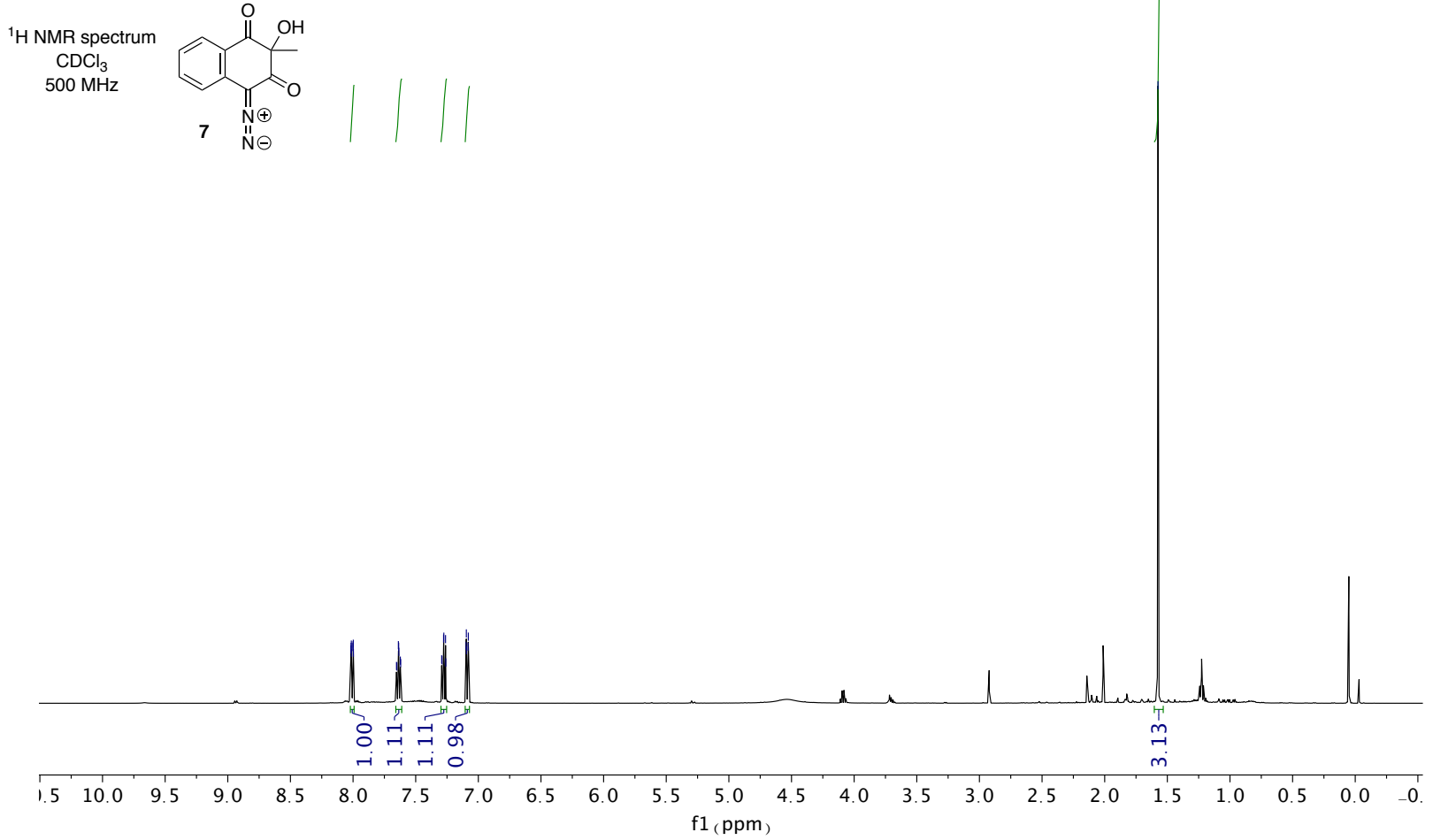

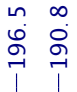

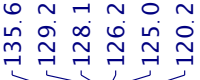

$\underset{\infty}{\infty}$

$\stackrel{\sim}{\sim} \stackrel{\infty}{\sim}$<smiles>CC1(O)C(=O)c2ccccc2C([N+](=O)[O-])C1(C)O</smiles>

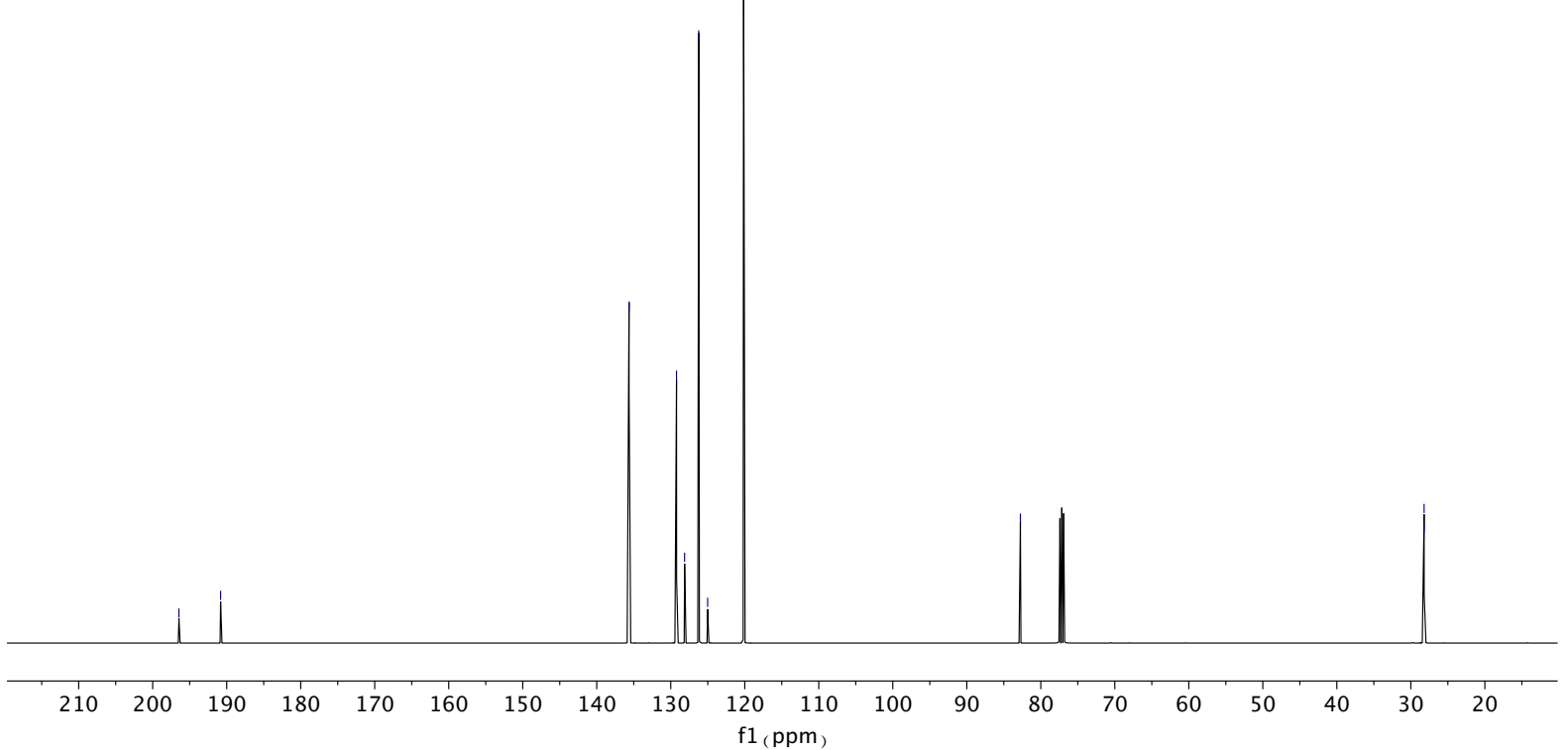




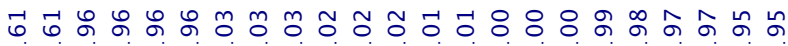

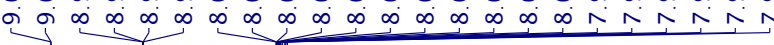
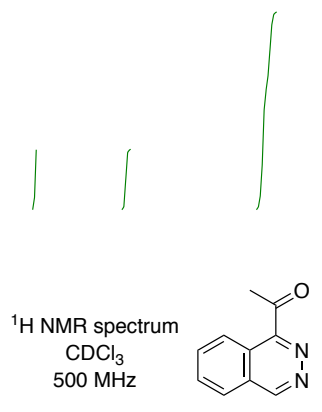

8
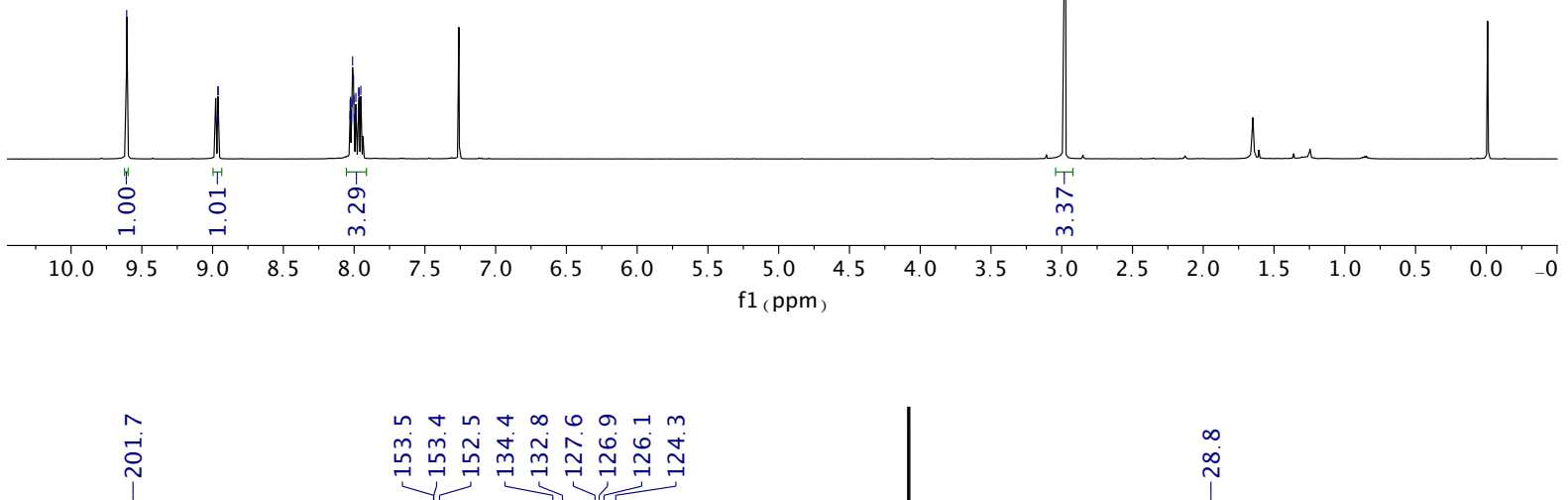

$\stackrel{\infty}{\infty}$

${ }^{13} \mathrm{C}\left\{{ }^{1} \mathrm{H}\right\}$ NMR spectrum

$\mathrm{CDCl}_{3}$

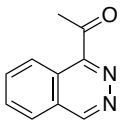

8

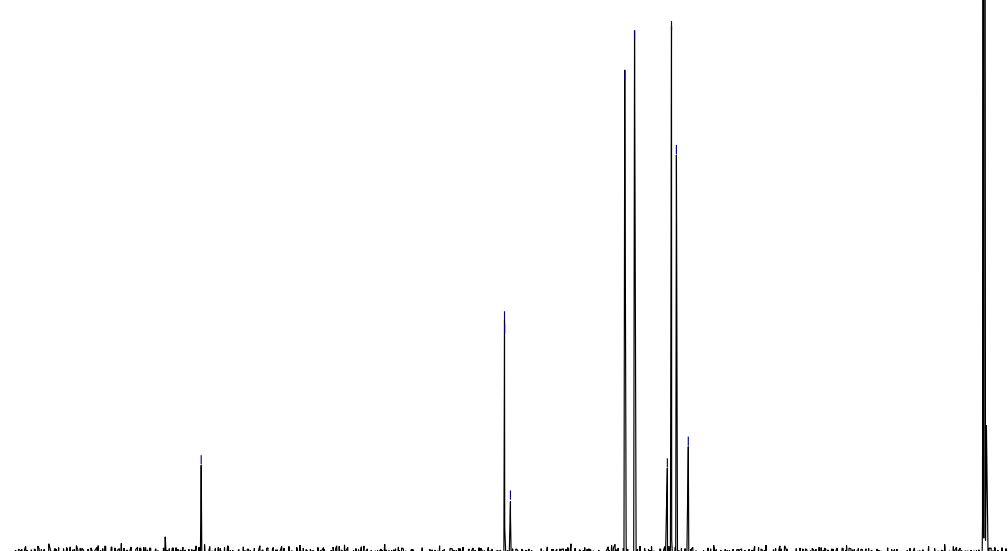

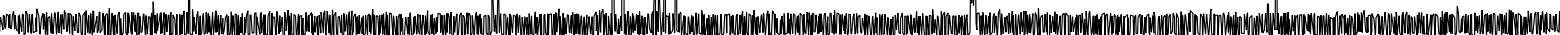

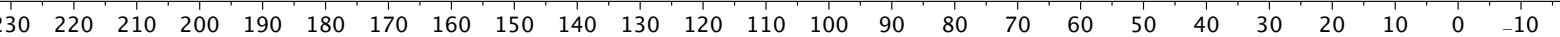
$\mathrm{f1}(\mathrm{ppm})$ 

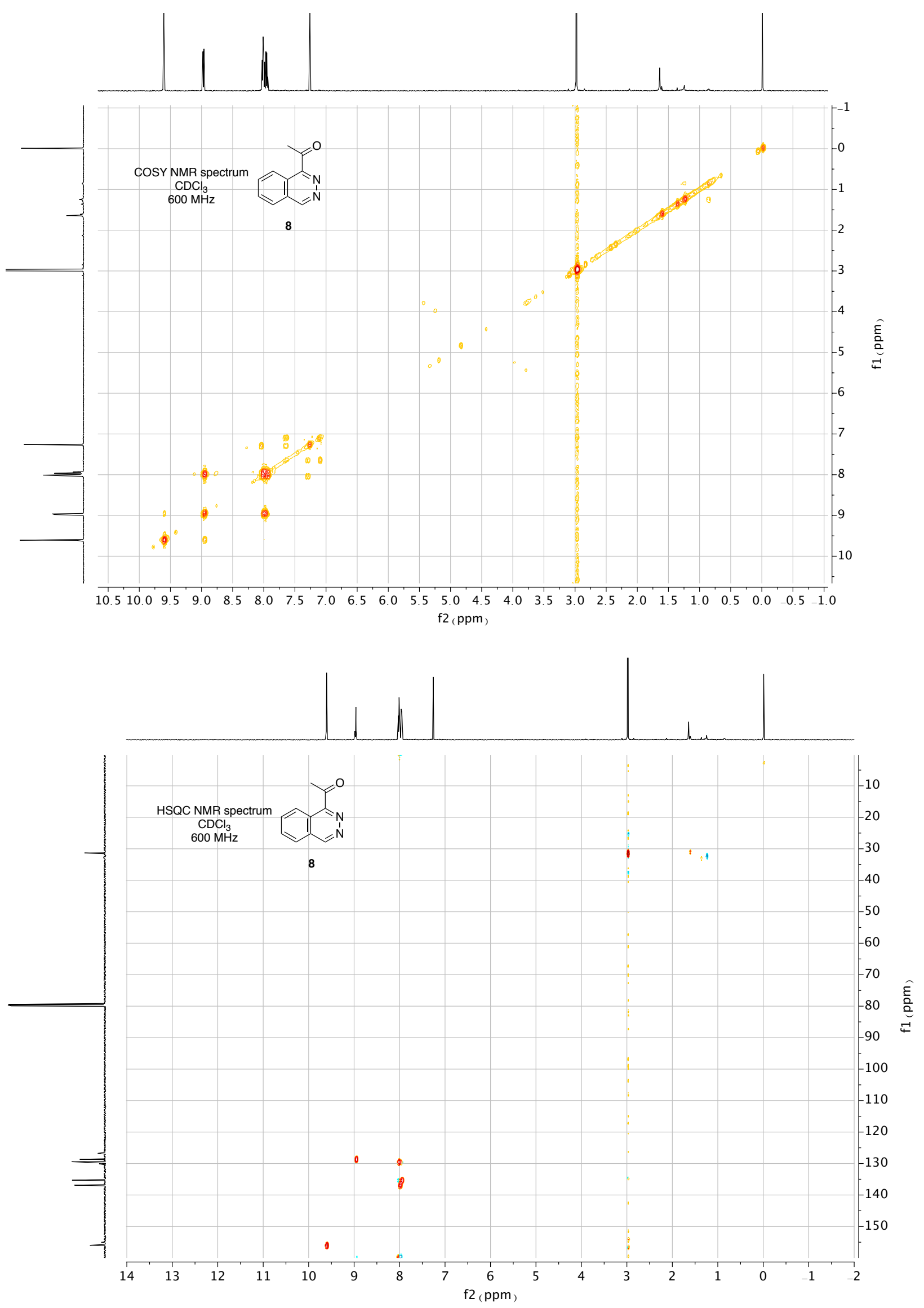


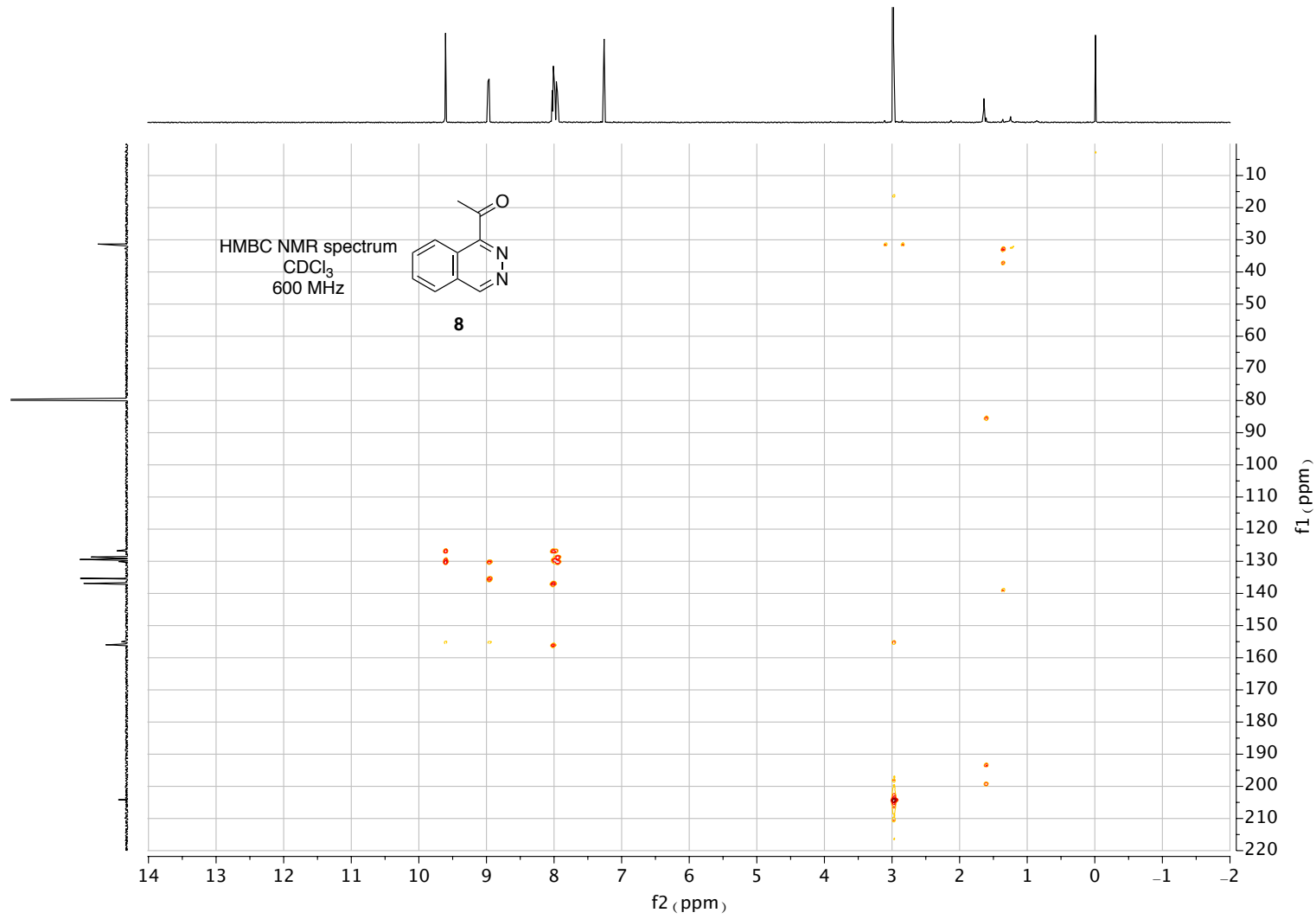



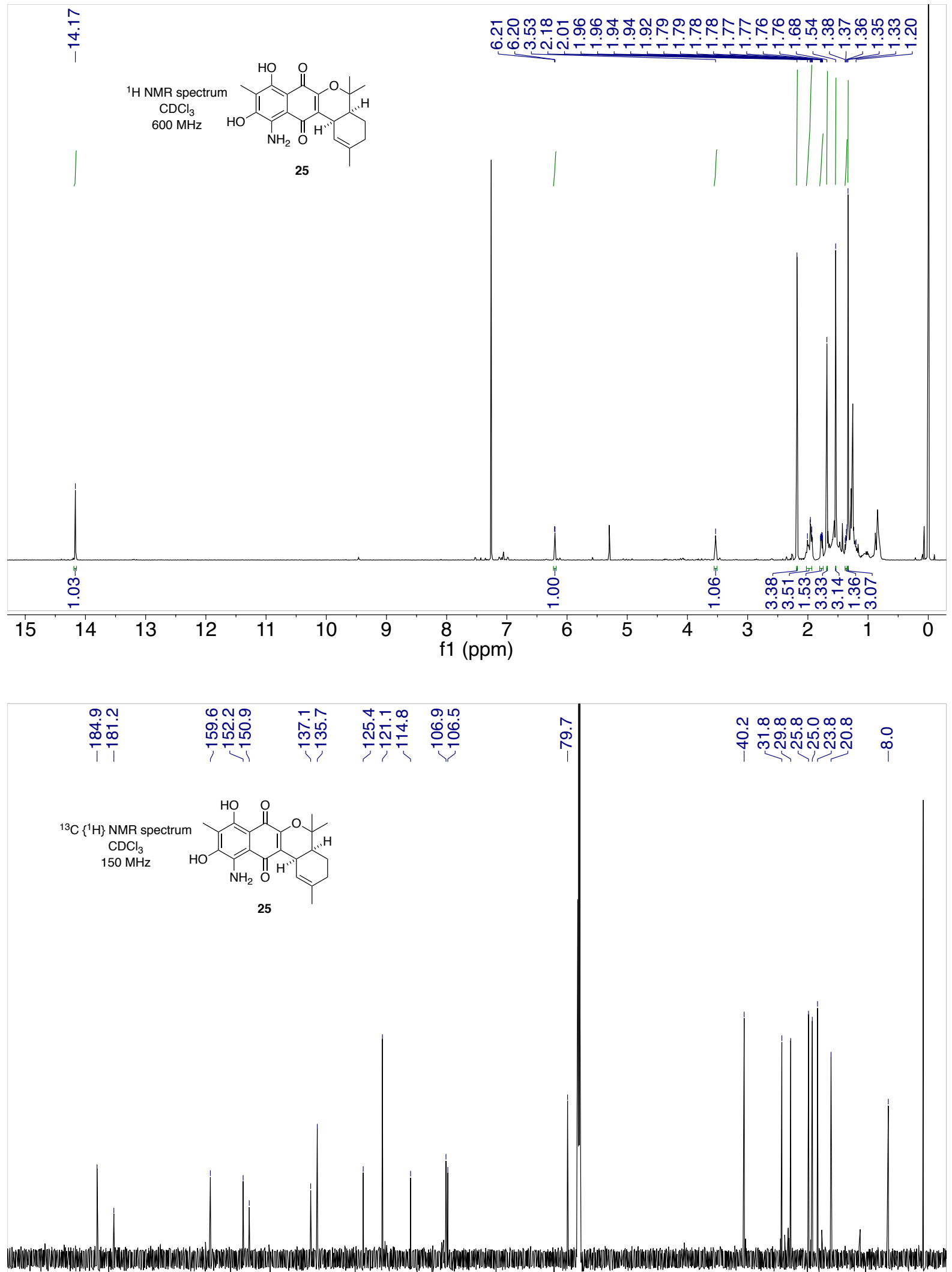

\begin{tabular}{lllllllllllllllllllllll}
\hline 200 & 190 & 180 & 170 & 160 & 150 & 140 & 130 & 120 & 110 & 100 & 90 & 80 & 70 & 60 & 50 & 40 & 30 & 20 & 10 & 0
\end{tabular} f1 (ppm) 

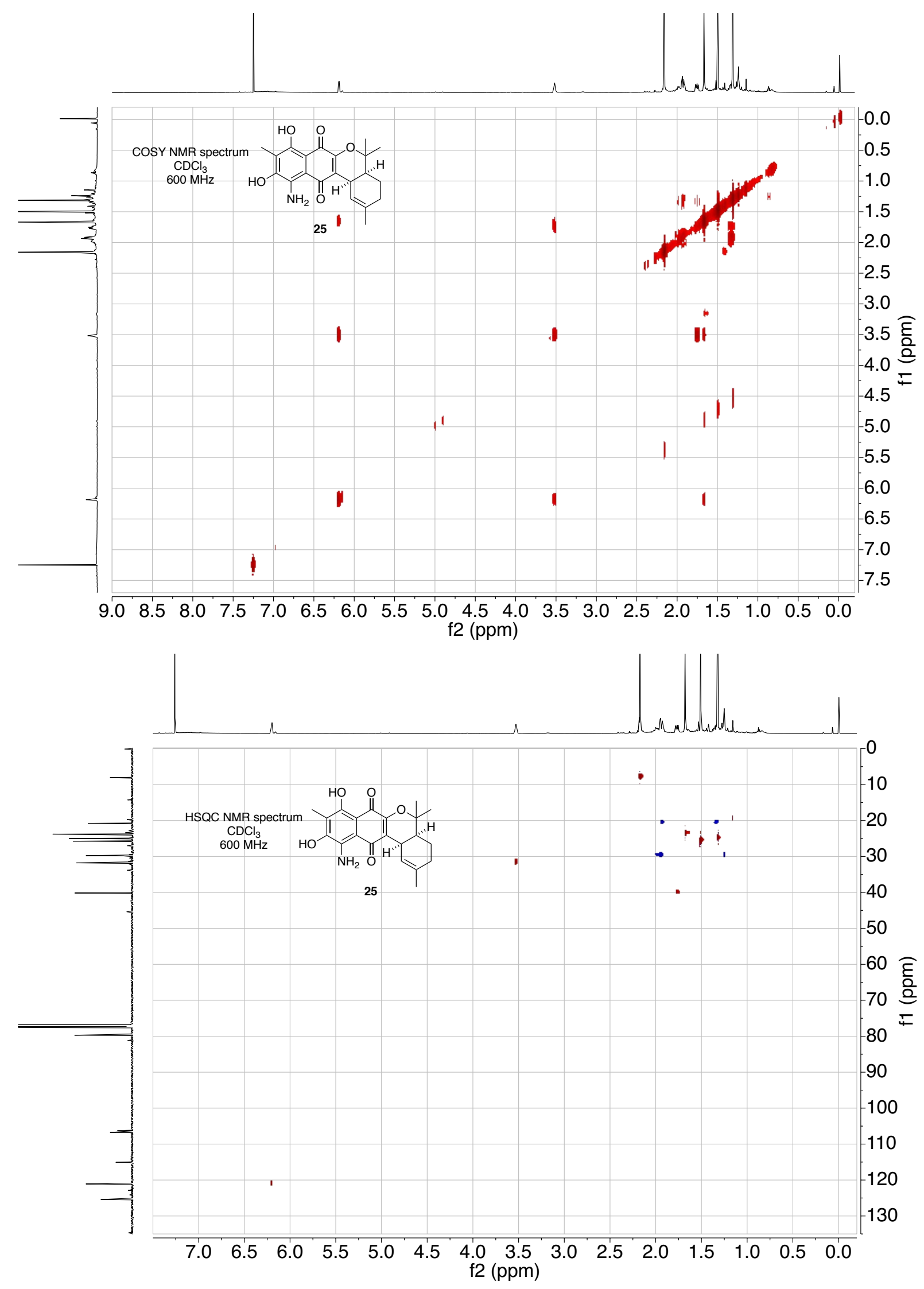


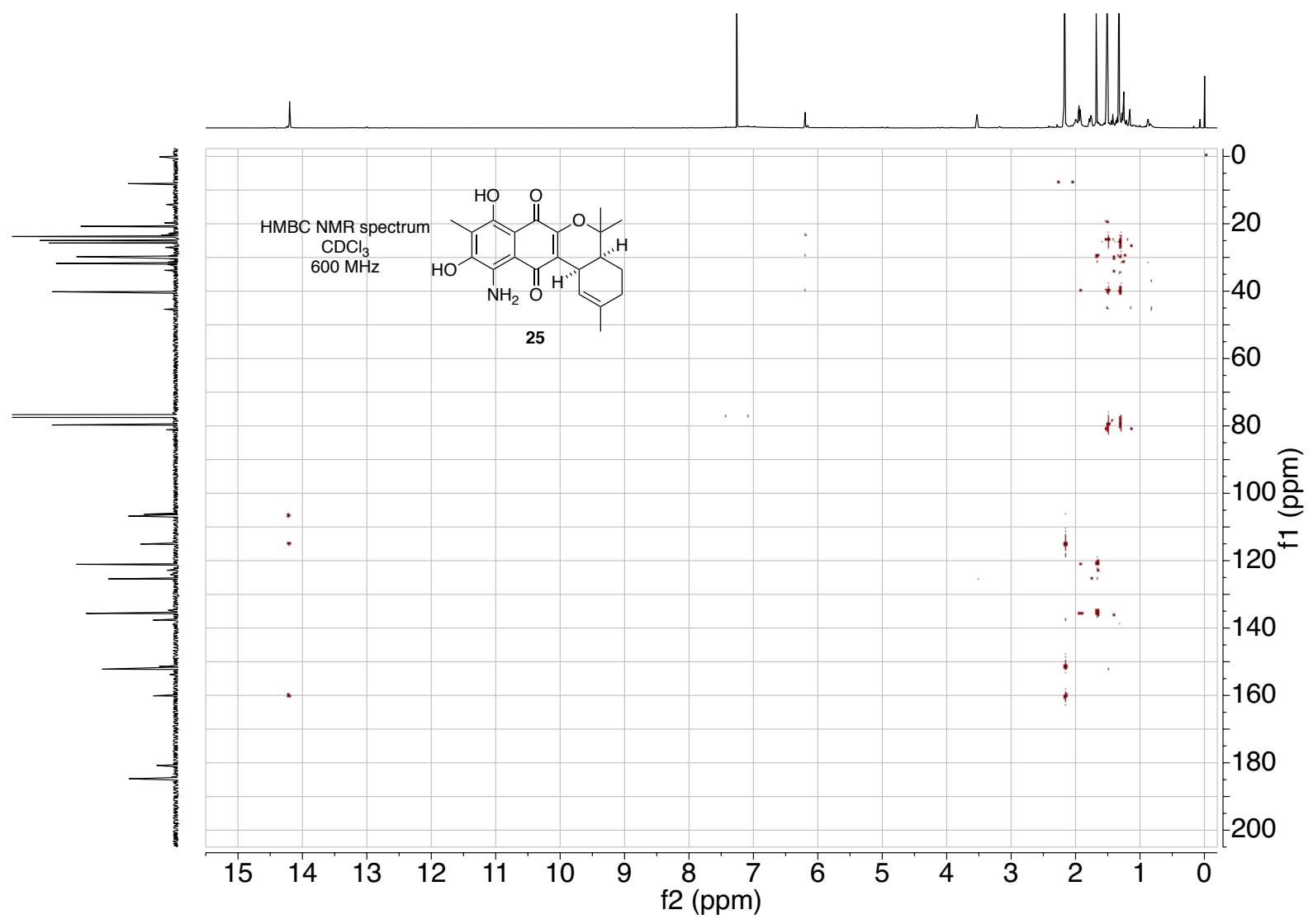



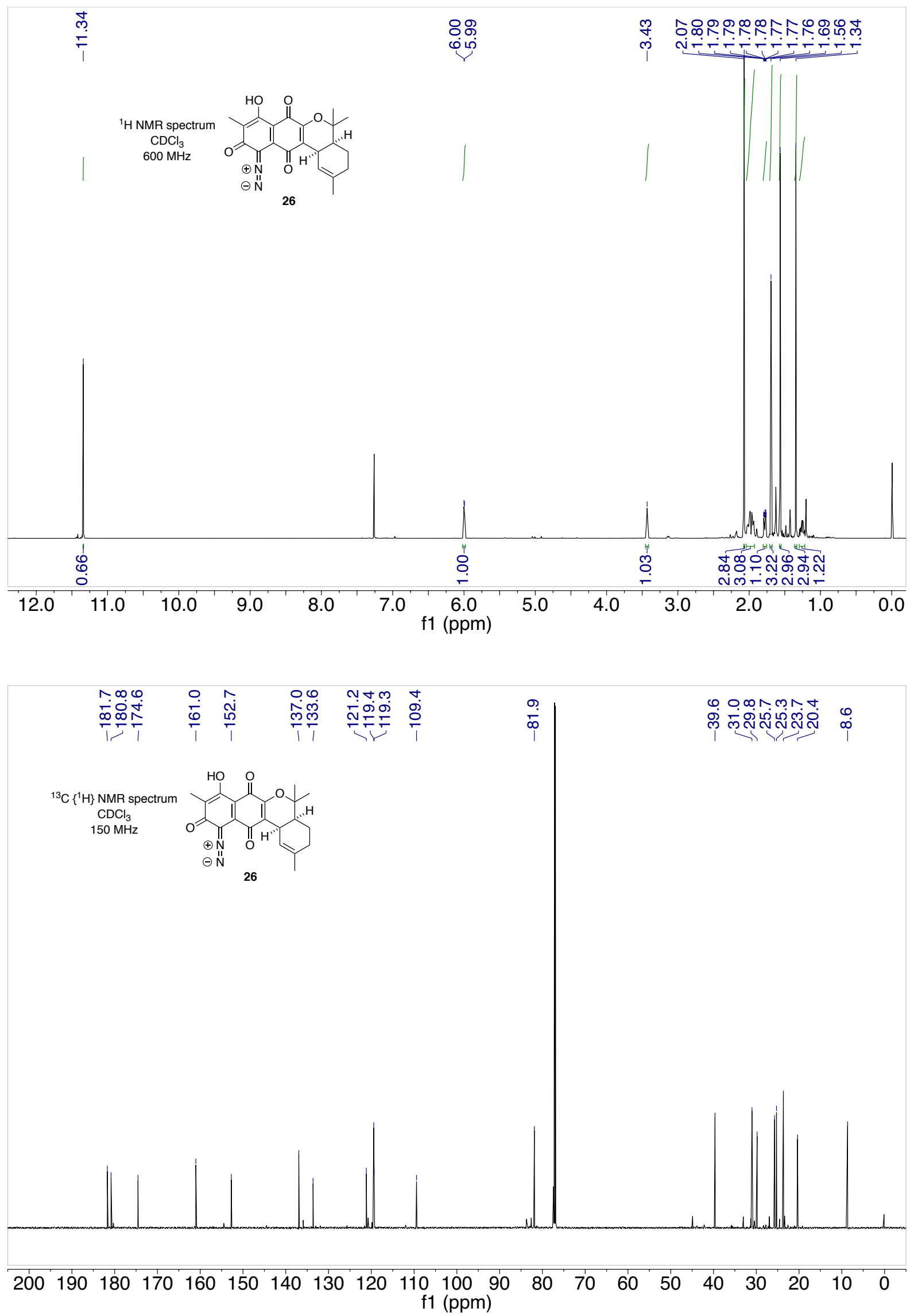

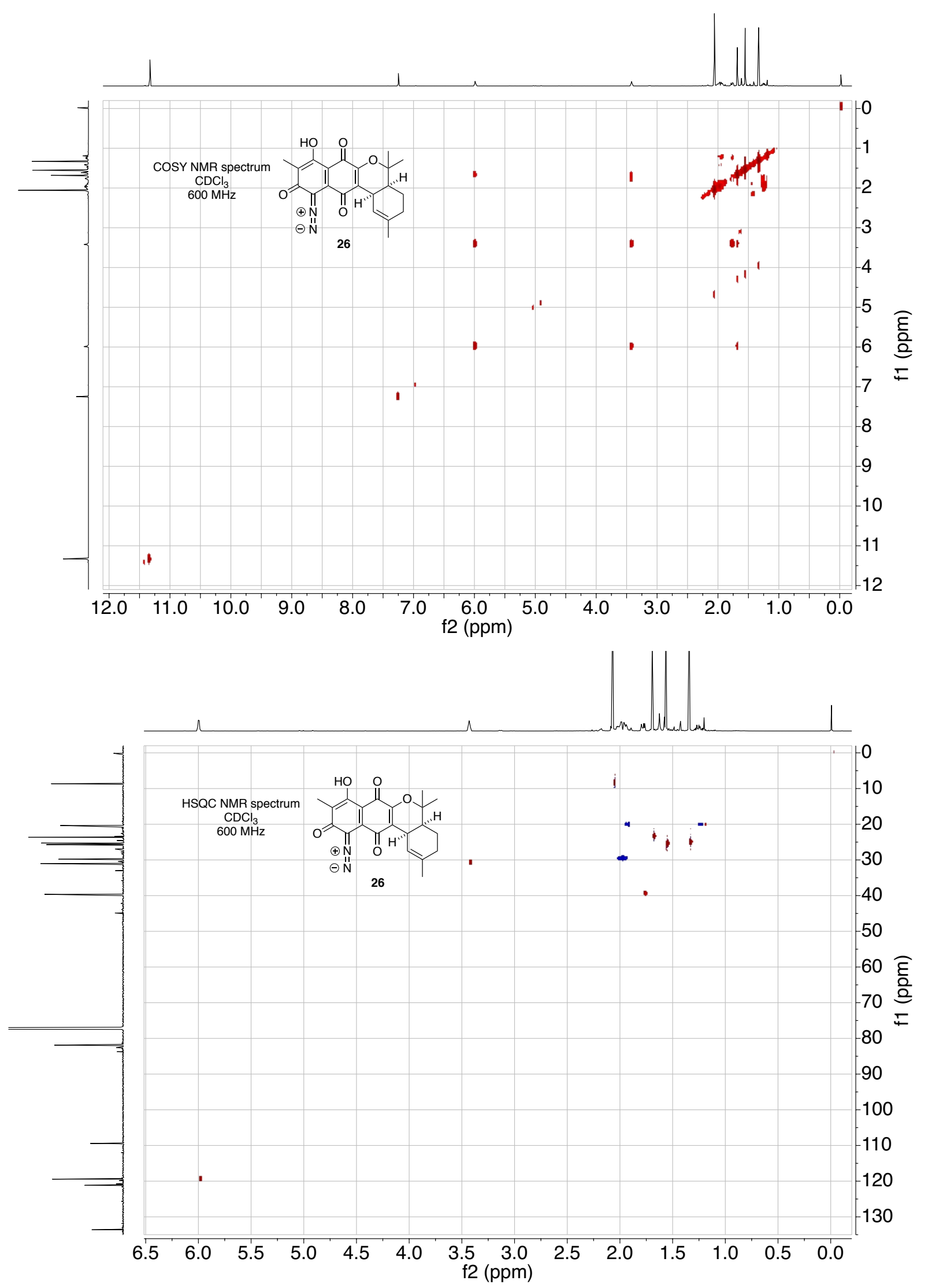

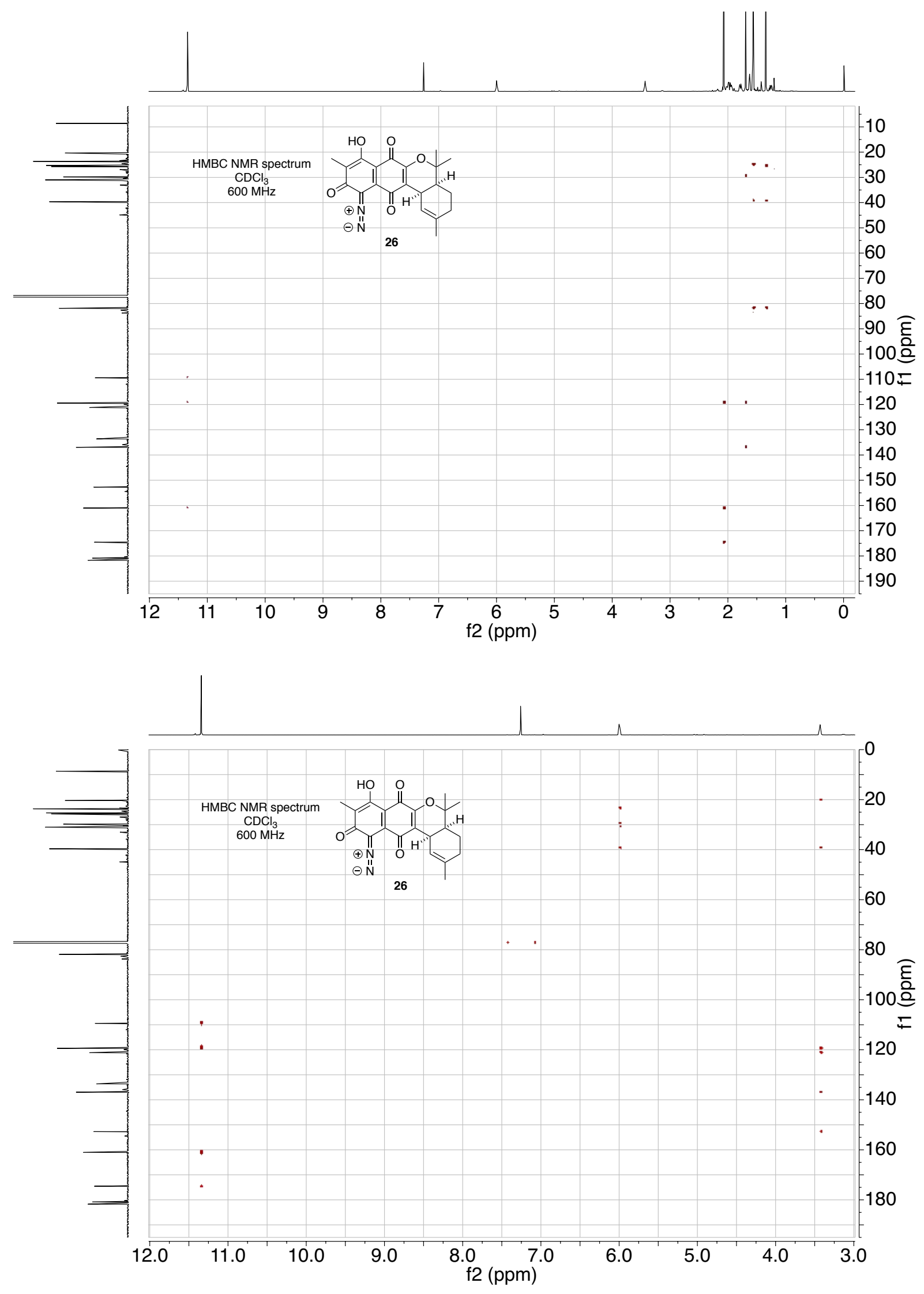

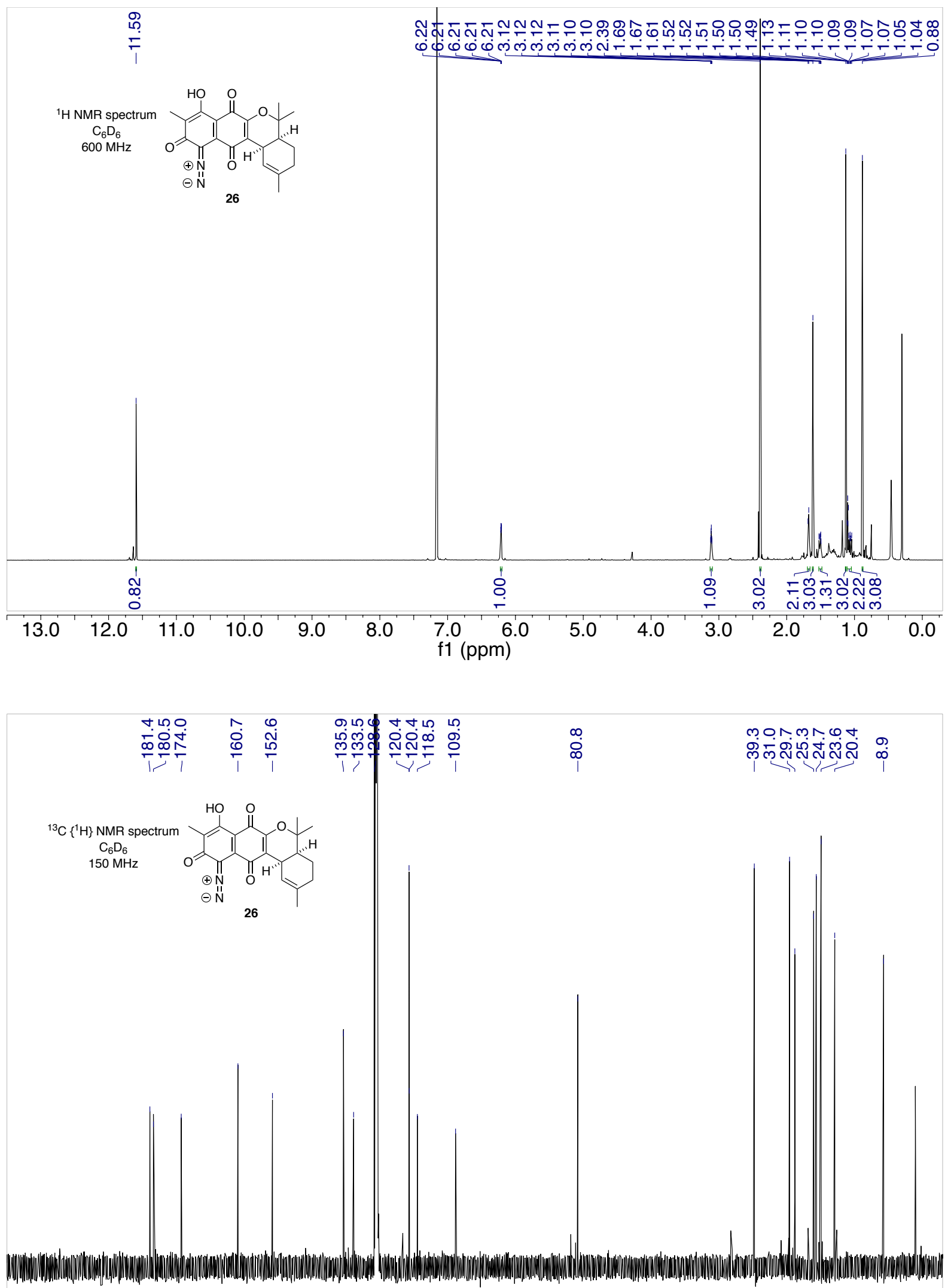

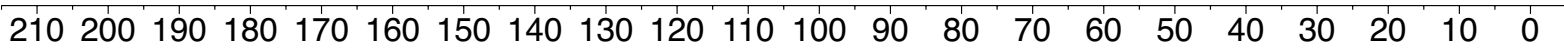
f1 (ppm) 


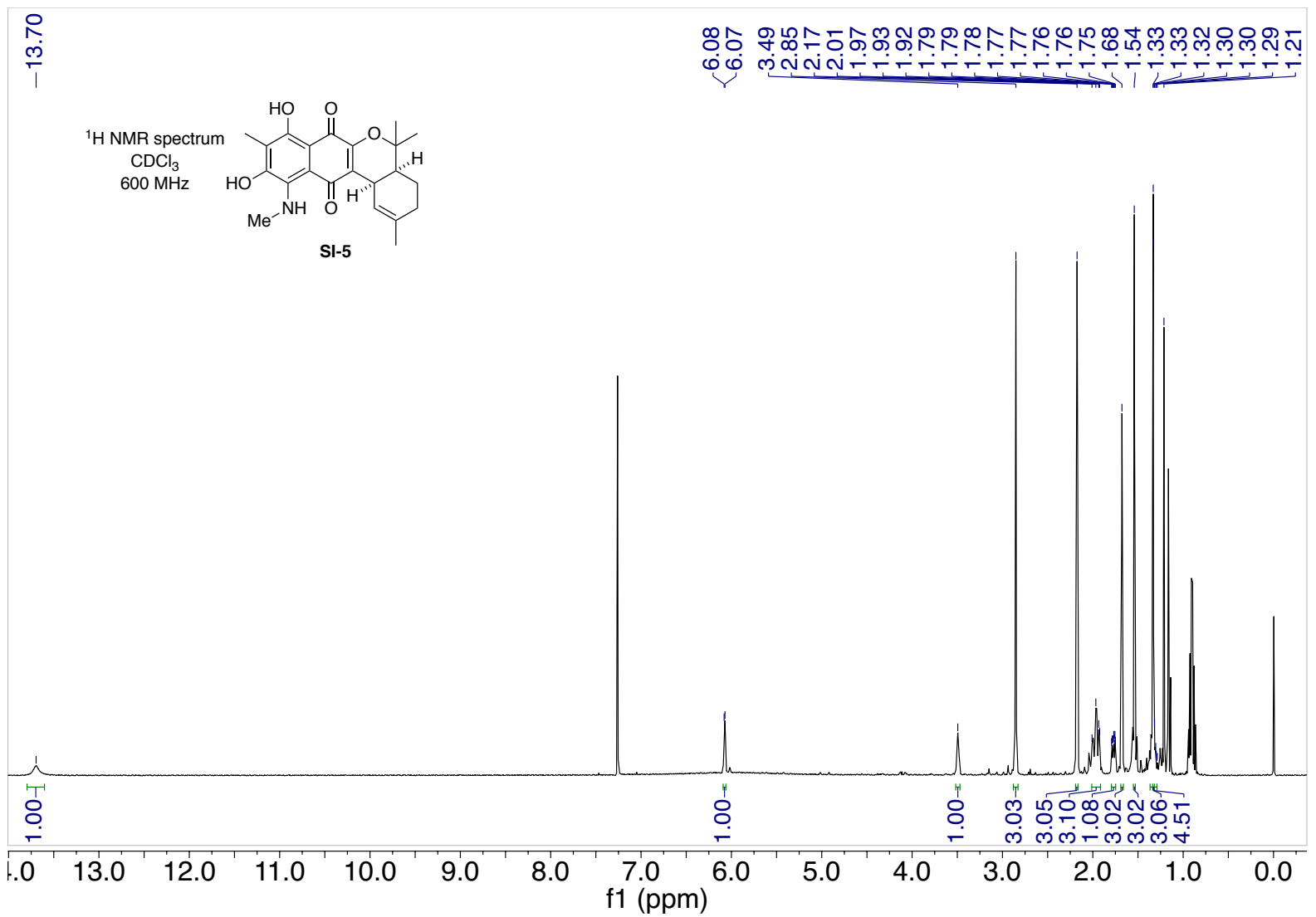

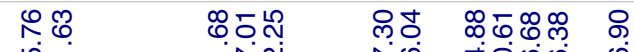

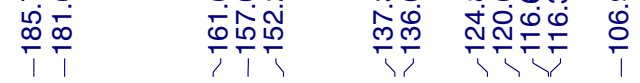

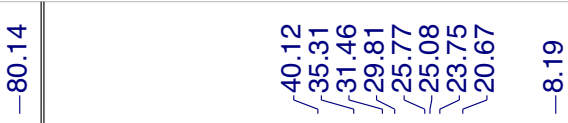

${ }^{13} \mathrm{C}\left\{{ }^{1} \mathrm{H}\right\} \mathrm{NMR}_{\mathrm{CDCl}_{3}}$

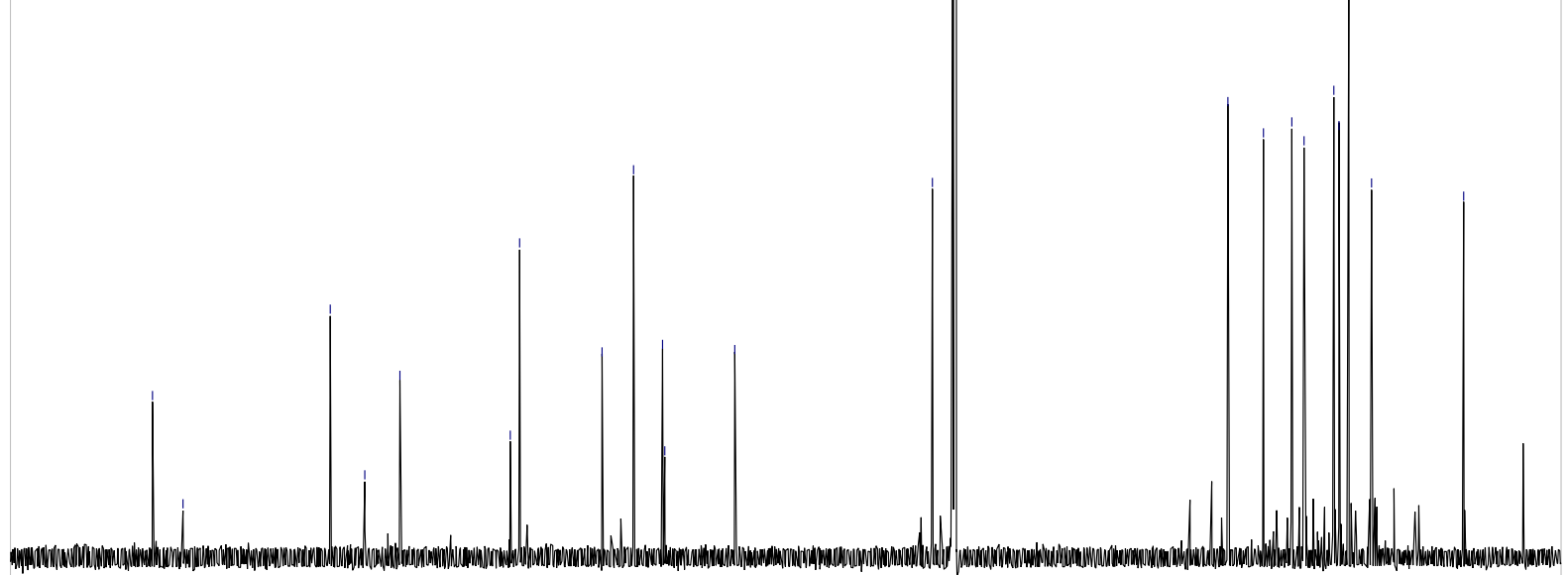

\begin{tabular}{llllllllllllllllllllll}
\hline 200 & 190 & 180 & 170 & 160 & 150 & 140 & 130 & 120 & 110 & 100 & 90 & 80 & 70 & 60 & 50 & 40 & 30 & 20 & 10 & 0
\end{tabular} f1 (ppm) 


\section{Selected IR Spectra}
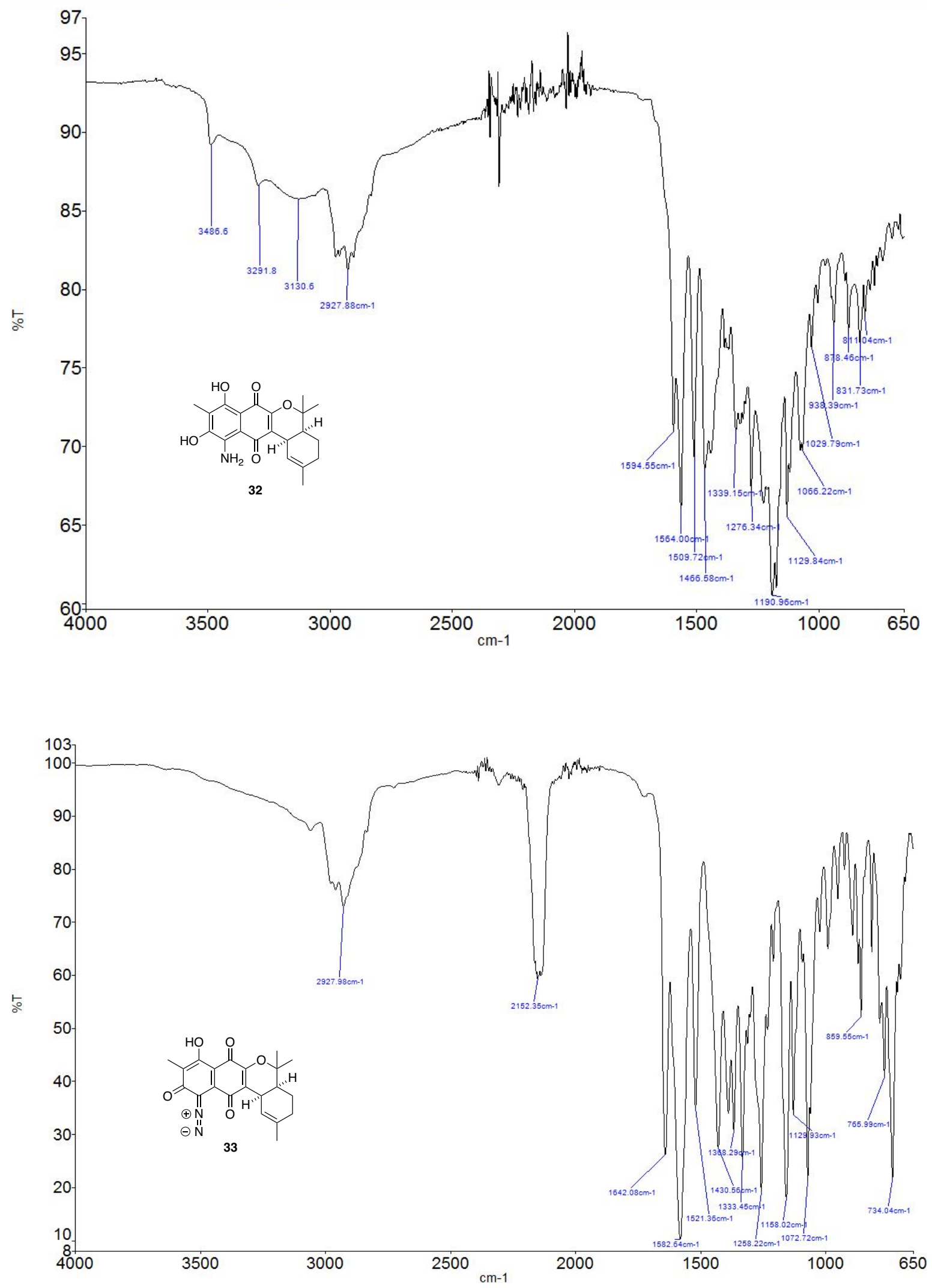


\section{Computational Studies}

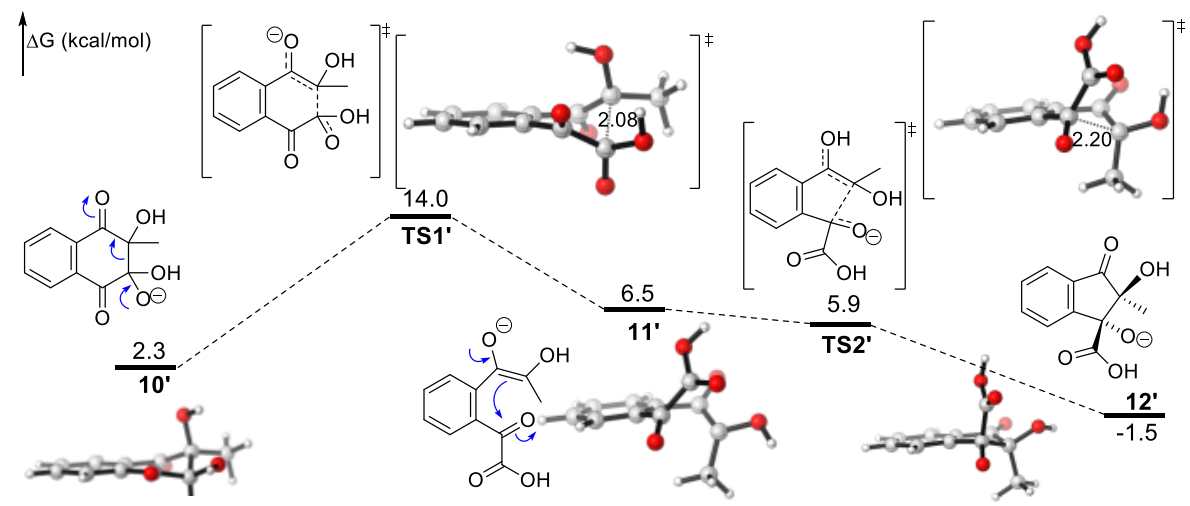

Figure S1. The Gibbs free energy profile of the Hooker reaction of 10' (diastereomer of 10). Energies are shown in $\mathrm{kcal} / \mathrm{mol}$, and bond lengths are given in $\AA$.

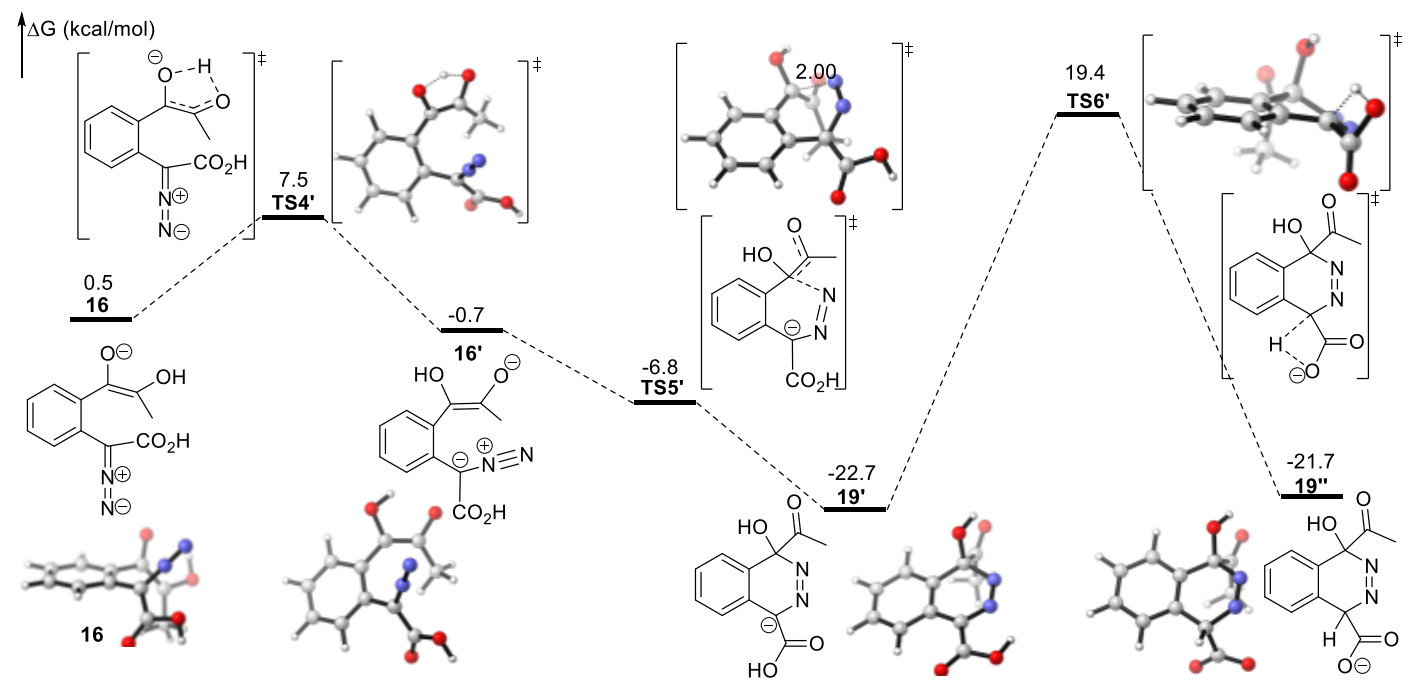

Figure S2. The Gibbs free energy profile of the diazo-Hooker reaction through 6-endo-cyclization. Energies are shown in $\mathrm{kcal} / \mathrm{mol}$, and bond lengths are given in $\AA$. 


\section{Cartesian coordinates for all optimized geometries}

10

Thermal correction to Gibbs Free Energy $=0.142127$

Sum of electronic and thermal Free Energies $=-800.781284$

E_sol $=-801.1275221$ Hartree

$\begin{array}{lrrr}\text { C } & 3.52245900 & 0.66767500 & 0.09466200 \\ \mathrm{C} & 2.32610800 & 1.38460700 & 0.07850000 \\ \mathrm{C} & 1.10016300 & 0.71150300 & 0.00034600 \\ \mathrm{C} & 1.08160800 & -0.69929200 & -0.06700600 \\ \mathrm{C} & 2.28826100 & -1.41103900 & -0.05468900 \\ \mathrm{C} & 3.50358700 & -0.73155600 & 0.02982400 \\ \mathrm{H} & 4.46920100 & 1.19657100 & 0.15749200 \\ \mathrm{H} & 2.33305500 & 2.46912500 & 0.12196700 \\ \mathrm{H} & 2.26566500 & -2.49523800 & -0.10071900 \\ \mathrm{H} & 4.43511900 & -1.29001000 & 0.04639500 \\ \mathrm{C} & -1.48390800 & -0.71811200 & 0.30277600 \\ \mathrm{C} & -0.17178000 & 1.47959200 & 0.00198300 \\ \mathrm{C} & -0.20490400 & -1.44203900 & -0.15690600 \\ 0 & -0.24210700 & -2.60862300 & -0.54427400 \\ \mathrm{C} & -1.46562900 & 0.71332500 & -0.32595900 \\ \mathrm{C} & -1.53869000 & -0.74808700 & 1.83246700 \\ \mathrm{H} & -2.47038100 & -0.28637400 & 2.16917400 \\ \mathrm{H} & -1.50434600 & -1.78319200 & 2.18935100 \\ \mathrm{H} & -0.69777800 & -0.20173400 & 2.27051300 \\ \mathrm{O} & -2.63105300 & -1.40196300 & -0.18632400 \\ \mathrm{H} & -2.69464300 & -1.16252200 & -1.13371600 \\ \mathrm{O} & -1.51548500 & 0.64214800 & -1.68703400 \\ 0 & -2.58723800 & 1.41344200 & 0.25163400 \\ \mathrm{H} & -2.54822200 & 2.32087700 & -0.10085300 \\ \mathrm{O} & -0.19053100 & 2.70412700 & 0.16154800\end{array}$

\section{TS1}

Thermal correction to Gibbs Free Energy $=0.141493$

Sum of electronic and thermal Free Energies $=-800.765193$ E_sol $=-801.1122737$ Hartree

$\begin{array}{lrrr}\text { C } & 3.52245900 & 0.66767500 & 0.09466200 \\ \text { C } & 2.32610800 & 1.38460700 & 0.07850000 \\ \text { C } & 1.10016300 & 0.71150300 & 0.00034600 \\ \text { C } & 1.08160800 & -0.69929200 & -0.06700600 \\ \text { C } & 2.28826100 & -1.41103900 & -0.05468900 \\ \text { C } & 3.50358700 & -0.73155600 & 0.02982400 \\ \text { H } & 4.46920100 & 1.19657100 & 0.15749200 \\ \text { H } & 2.33305500 & 2.46912500 & 0.12196700 \\ \text { H } & 2.26566500 & -2.49523800 & -0.10071900 \\ \text { H } & 4.43511900 & -1.29001000 & 0.04639500 \\ \text { C } & -1.48390800 & -0.71811200 & 0.30277600 \\ \text { C } & -0.17178000 & 1.47959200 & 0.00198300\end{array}$




$\begin{array}{lrrr}\mathrm{C} & -0.20490400 & -1.44203900 & -0.15690600 \\ 0 & -0.24210700 & -2.60862300 & -0.54427400 \\ \mathrm{C} & -1.46562900 & 0.71332500 & -0.32595900 \\ \mathrm{C} & -1.53869000 & -0.74808700 & 1.83246700 \\ \mathrm{H} & -2.47038100 & -0.28637400 & 2.16917400 \\ \mathrm{H} & -1.50434600 & -1.78319200 & 2.18935100 \\ \mathrm{H} & -0.69777800 & -0.20173400 & 2.27051300 \\ \mathrm{O} & -2.63105300 & -1.40196300 & -0.18632400 \\ \mathrm{H} & -2.69464300 & -1.16252200 & -1.13371600 \\ 0 & -1.51548500 & 0.64214800 & -1.68703400 \\ 0 & -2.58723800 & 1.41344200 & 0.25163400 \\ \mathrm{H} & -2.54822200 & 2.32087700 & -0.10085300 \\ 0 & -0.19053100 & 2.70412700 & 0.16154800\end{array}$

\section{1}

Thermal correction to Gibbs Free Energy $=0.140142$

Sum of electronic and thermal Free Energies $=-800.774023$ E_sol $=-801.1209822$ Hartree

$\begin{array}{lrrr}\text { C } & 3.41818000 & 0.66640300 & 0.12014000 \\ \mathrm{C} & 2.21651900 & 1.35661800 & 0.25526000 \\ \mathrm{C} & 1.00150400 & 0.66780000 & 0.09467600 \\ \mathrm{C} & 0.99770900 & -0.72715000 & -0.10679700 \\ \mathrm{C} & 2.21354800 & -1.40666500 & -0.28098200 \\ \mathrm{C} & 3.41568700 & -0.71176400 & -0.16439000 \\ \mathrm{H} & 4.36170800 & 1.19676500 & 0.21708300 \\ \mathrm{H} & 2.20844300 & 2.42694700 & 0.44198000 \\ \mathrm{H} & 2.20889400 & -2.47752900 & -0.46587800 \\ \mathrm{H} & 4.35880800 & -1.24042800 & -0.27563700 \\ \mathrm{C} & -1.27935700 & -1.01586600 & 0.83126700 \\ \mathrm{C} & -0.29184700 & 1.38282500 & 0.12421000 \\ \mathrm{C} & -0.31545000 & -1.39903700 & -0.12114800 \\ \mathrm{O} & -0.62923400 & -2.22883200 & -1.06691800 \\ \mathrm{C} & -1.29023800 & 1.06699700 & -0.96400500 \\ \mathrm{C} & -1.10834400 & -0.46991500 & 2.20930400 \\ \mathrm{H} & -1.72964900 & 0.42312400 & 2.36901200 \\ \mathrm{H} & -1.42547200 & -1.22715300 & 2.94009600 \\ \mathrm{H} & -0.07043000 & -0.20689500 & 2.41832400 \\ \mathrm{O} & -2.54991500 & -1.51805100 & 0.61915300 \\ \mathrm{H} & -2.50929900 & -1.98263900 & -0.24580600 \\ \mathrm{O} & -1.01060900 & 0.63305700 & -2.08453000 \\ \mathrm{O} & -2.54581600 & 1.46680400 & -0.63869500 \\ \mathrm{H} & -3.12841100 & 1.29143500 & -1.40333100 \\ \mathrm{O} & -0.53218200 & 2.31388500 & 0.92335900\end{array}$

\section{TS2}

Thermal correction to Gibbs Free Energy $=0.140428$

Sum of electronic and thermal Free Energies $=-800.757128$ E_sol $=-801.1042993$ Hartree 


$\begin{array}{lrrr}\text { C } & 3.14723300 & -1.20152000 & -0.06964900 \\ \mathrm{C} & 1.84120100 & -1.55383200 & 0.29037400 \\ \mathrm{C} & 0.86733600 & -0.55293100 & 0.34322300 \\ \mathrm{C} & 1.18726000 & 0.76931700 & 0.00988400 \\ \mathrm{C} & 2.49463700 & 1.12916500 & -0.32253600 \\ \mathrm{C} & 3.47400400 & 0.13078800 & -0.36615700 \\ \mathrm{H} & 3.91890000 & -1.96606200 & -0.10931400 \\ \mathrm{H} & 1.59230900 & -2.58213200 & 0.53908900 \\ \mathrm{H} & 2.73853300 & 2.16015000 & -0.56577900 \\ \mathrm{H} & 4.49450600 & 0.38651200 & -0.63903100 \\ \mathrm{C} & -1.16664600 & 1.17675200 & -0.57587000 \\ \mathrm{C} & -0.54769400 & -0.70166000 & 0.85281300 \\ \mathrm{C} & -0.01583700 & 1.64485200 & 0.11020300 \\ \mathrm{O} & -0.09022600 & 2.56334500 & 0.99706500 \\ \mathrm{C} & -1.54731600 & -1.52689800 & 0.09249200 \\ \mathrm{C} & -1.20077800 & 0.66798500 & -1.98365300 \\ \mathrm{H} & -2.04845100 & -0.00871000 & -2.14512700 \\ \mathrm{H} & -1.31870000 & 1.51351400 & -2.67741800 \\ \mathrm{H} & -0.27960100 & 0.14257100 & -2.24190000 \\ \mathrm{O} & -2.37524000 & 1.74158100 & -0.18960200 \\ \mathrm{H} & -2.20500300 & 2.23894700 & 0.63619300 \\ \mathrm{O} & -2.73351400 & -1.61840700 & 0.40207100 \\ \mathrm{O} & -1.01997000 & -2.17707000 & -0.96723200 \\ \mathrm{H} & -1.73341400 & -2.66784600 & -1.42279700 \\ \mathrm{O} & -0.82598300 & -0.39808000 & 2.05011400\end{array}$

12

Thermal correction to Gibbs Free Energy $=0.142691$

Sum of electronic and thermal Free Energies $=-800.788315$ E_sol $=-801.1358703$ Hartree

$\begin{array}{lrrr}\text { C } & -2.91515700 & -1.55381400 & -0.14354900 \\ \mathrm{C} & -1.53646800 & -1.68279600 & -0.33094100 \\ \mathrm{C} & -0.73693500 & -0.54301100 & -0.22741700 \\ \mathrm{C} & -1.32244600 & 0.69609300 & 0.05982600 \\ \mathrm{C} & -2.70643400 & 0.83479100 & 0.24240500 \\ \mathrm{C} & -3.50085500 & -0.30388300 & 0.14029900 \\ \mathrm{H} & -3.55023600 & -2.43284700 & -0.21822500 \\ \mathrm{H} & -1.09532000 & -2.65093300 & -0.55334000 \\ \mathrm{H} & -3.13813500 & 1.80769300 & 0.46145300 \\ \mathrm{H} & -4.57594600 & -0.23411600 & 0.28023900 \\ \mathrm{C} & 1.10078600 & 1.07365900 & 0.00656200 \\ \mathrm{C} & 0.75620500 & -0.42292100 & -0.52212700 \\ \mathrm{C} & -0.29290200 & 1.73458500 & 0.11275300 \\ 0 & -0.46732100 & 2.94878900 & 0.23476500 \\ \mathrm{C} & 1.60334900 & -1.44836400 & 0.24955900 \\ \mathrm{C} & 1.80466100 & 1.19920000 & 1.35550700 \\ \mathrm{H} & 2.77881200 & 0.69899500 & 1.32318800 \\ \mathrm{H} & 1.96496500 & 2.25995400 & 1.57782500\end{array}$




$\begin{array}{rrrr}\mathrm{H} & 1.20780800 & 0.76316400 & 2.16219200 \\ \mathrm{O} & 1.88231200 & 1.68729500 & -1.00187300 \\ \mathrm{H} & 1.74576800 & 1.03012800 & -1.75625800 \\ \mathrm{O} & 2.67216900 & -1.89101200 & -0.14770700 \\ 0 & 1.07853500 & -1.80355700 & 1.44081300 \\ \mathrm{H} & 1.69574300 & -2.41327700 & 1.89428300 \\ 0 & 1.01476900 & -0.48276400 & -1.87957500\end{array}$

15

Thermal correction to Gibbs Free Energy $=0.145549$

Sum of electronic and thermal Free Energies $=-835.002676$

E_sol $=-835.3576687$ Hartree

$\begin{array}{lrrr}\text { C } & -3.53378700 & 0.54060600 & -0.03751000 \\ \text { C } & -2.33251100 & 1.23992600 & -0.02788900 \\ \text { C } & -1.09877200 & 0.55331000 & 0.01016200 \\ \text { C } & -1.11550400 & -0.86634000 & 0.03741600 \\ \text { C } & -2.34374700 & -1.55410600 & 0.04535500 \\ \text { C } & -3.54873100 & -0.86471800 & 0.00280100 \\ \text { H } & -4.46985100 & 1.09203600 & -0.07039400 \\ \text { H } & -2.34625300 & 2.32643800 & -0.04108000 \\ \text { H } & -2.33120400 & -2.63970000 & 0.06625000 \\ \text { H } & -4.49110100 & -1.40449500 & -0.00387800 \\ \text { C } & 1.43348800 & -0.90758900 & -0.36174500 \\ \text { C } & 1.53302700 & 0.50899500 & 0.32619300 \\ \text { C } & 0.17775500 & 1.23427400 & 0.04639300 \\ \text { C } & 0.14345800 & -1.63361200 & 0.05630200 \\ \text { O } & 0.17173700 & -2.82854900 & 0.37322200 \\ \text { O } & 1.29719900 & -0.66886000 & -1.77689100 \\ \text { H } & 1.90304800 & 0.07398200 & -1.97239500 \\ \text { O } & 2.58686600 & 1.20971000 & -0.07194200 \\ \text { C } & 2.67356200 & -1.75024400 & -0.09475800 \\ \text { H } & 3.56094300 & -1.19653700 & -0.41347800 \\ \text { H } & 2.77197100 & -1.99458700 & 0.96631400 \\ \text { H } & 2.61565600 & -2.68227700 & -0.66441900 \\ \text { N } & 0.24049000 & 2.51848600 & -0.10248900 \\ \text { N } & 0.37951400 & 3.64939100 & -0.24351000 \\ \text { O } & 1.54800500 & 0.18897200 & 1.76497700 \\ \text { H } & 1.68689200 & 1.03681100 & 2.22382100\end{array}$

\section{TS3}

Thermal correction to Gibbs Free Energy $=0.144175$

Sum of electronic and thermal Free Energies $=-834.979236$

E_sol $=-835.3349593$ Hartree

$\begin{array}{lrrr}\text { C } & -3.52750600 & 0.42324900 & -0.01634000 \\ \mathrm{C} & -2.34749400 & 1.15640600 & -0.14893600 \\ \mathrm{C} & -1.09766000 & 0.51240800 & -0.12556500 \\ \mathrm{C} & -1.04806100 & -0.89423700 & 0.02688600 \\ \mathrm{C} & -2.24311100 & -1.61746400 & 0.12239600\end{array}$




$\begin{array}{rrrr}\text { C } & -3.48026400 & -0.96941500 & 0.11695700 \\ \mathrm{C} & 1.35081700 & -1.07593900 & 0.70341600 \\ \mathrm{C} & 1.48514000 & 0.68860000 & -0.65957200 \\ \mathrm{C} & 0.15880400 & 1.26241300 & -0.24221300 \\ \mathrm{C} & 0.26446300 & -1.61154500 & -0.01241400 \\ \mathrm{O} & 0.38825600 & -2.59826100 & -0.82313300 \\ \mathrm{O} & 1.06698000 & -0.39378900 & 1.90431100 \\ \mathrm{O} & 1.40816700 & 0.12077400 & -1.92993800 \\ \mathrm{O} & 2.56331600 & 1.20852000 & -0.27528700 \\ \mathrm{C} & 2.71921700 & -1.68006700 & 0.63059300 \\ \mathrm{~N} & 0.20678700 & 2.49477500 & 0.16707700 \\ \mathrm{~N} & 0.28365900 & 3.58344700 & 0.52407300 \\ \mathrm{H} & -4.48355200 & 0.93990600 & -0.03199900 \\ \mathrm{H} & -2.39851600 & 2.23393900 & -0.28403500 \\ \mathrm{H} & -2.19210700 & -2.69869000 & 0.22154500 \\ \mathrm{H} & -4.39782500 & -1.54367400 & 0.21213800 \\ \mathrm{H} & 1.68251300 & 0.36107400 & 1.97121400 \\ \mathrm{H} & 2.29939700 & -0.20953400 & -2.15169400 \\ \mathrm{H} & 3.46963000 & -0.93677100 & 0.92670500 \\ \mathrm{H} & 2.81944500 & -2.54335800 & 1.30540900 \\ \mathrm{H} & 2.94807000 & -2.01484800 & -0.38622300\end{array}$

16

Thermal correction to Gibbs Free Energy $=0.142674$

Sum of electronic and thermal Free Energies $=-834.998358$ E_sol $=-835.353935$ Hartree

$\begin{array}{lrrr}\text { C } & -2.43620400 & -2.40544500 & 0.00154500 \\ \mathrm{C} & -1.06960200 & -2.21835900 & 0.20223800 \\ \mathrm{C} & -0.51934000 & -0.92443400 & 0.23237200 \\ \mathrm{C} & -1.34941800 & 0.20676800 & 0.04992300 \\ \mathrm{C} & -2.73000800 & -0.00751300 & -0.11148500 \\ \mathrm{C} & -3.27264800 & -1.29156800 & -0.14642300 \\ \mathrm{C} & 0.11077300 & 2.05351400 & -0.79179000 \\ \mathrm{C} & 1.96762800 & -1.51329500 & -0.22852500 \\ \mathrm{C} & 0.93752600 & -0.78497900 & 0.48146400 \\ \mathrm{C} & -0.84095300 & 1.61442300 & 0.07629100 \\ \text { O } & -1.37232500 & 2.44264900 & 0.99612700 \\ \text { O } & 0.52694600 & 3.39146300 & -0.64488900 \\ \text { O } & 3.22863700 & -1.22617900 & 0.19739600 \\ \text { O } & 1.74476500 & -2.31415800 & -1.13721900 \\ \mathrm{C} & 0.83062300 & 1.33987500 & -1.88554700 \\ \mathrm{~N} & 1.34605200 & 0.10402000 & 1.36354300 \\ \mathrm{~N} & 1.69153800 & 0.87369300 & 2.12322000 \\ \mathrm{H} & -2.84736500 & -3.41111000 & -0.02137200 \\ \mathrm{H} & -0.42002300 & -3.07581800 & 0.35087500 \\ \mathrm{H} & -3.37777400 & 0.85686300 & -0.23355900 \\ \mathrm{H} & -4.34138200 & -1.42566100 & -0.29366700 \\ \mathrm{H} & -0.00810100 & 3.72225200 & 0.10911300\end{array}$




$\begin{array}{rrrr}\mathrm{H} & 3.86015100 & -1.73926300 & -0.34371500 \\ \mathrm{H} & 1.89157700 & 1.17218900 & -1.63920800 \\ \mathrm{H} & 0.37994600 & 0.36579000 & -2.09208200 \\ \mathrm{H} & 0.80539400 & 1.92665400 & -2.81542600\end{array}$

\section{TS4}

Thermal correction to Gibbs Free Energy $=0.142631$

Sum of electronic and thermal Free Energies $=-834.984996$

E_sol $=-835.3397922$ Hartree

$\begin{array}{lrrr}\mathrm{C} & -1.13140800 & 3.18666300 & 0.05010400 \\ \mathrm{C} & -1.54790900 & 1.87066000 & -0.08927100 \\ \mathrm{C} & -0.62692900 & 0.79429500 & -0.13219300 \\ \mathrm{C} & 0.77195300 & 1.07970700 & -0.06955100 \\ \mathrm{C} & 1.15739600 & 2.43788000 & 0.02709000 \\ \mathrm{C} & 0.24006500 & 3.47586800 & 0.11037700 \\ \mathrm{H} & -1.86878900 & 3.98395500 & 0.09449500 \\ \mathrm{H} & -2.60712100 & 1.65934500 & -0.17914400 \\ \mathrm{H} & 2.21970000 & 2.65874400 & 0.05622200 \\ \mathrm{H} & 0.58422800 & 4.50137100 & 0.21754200 \\ \mathrm{C} & 1.95337900 & -1.03930100 & 0.60336600 \\ \mathrm{C} & -2.49199900 & -1.01463700 & 0.12042700 \\ \mathrm{C} & -1.20171000 & -0.55171600 & -0.34248900 \\ \mathrm{C} & 1.91839000 & 0.10438500 & -0.16140000 \\ \text { O } & 2.93175200 & 0.46553400 & -0.94369500 \\ \mathrm{O} & 0.96029100 & -1.38273500 & 1.53639400 \\ \mathrm{H} & 0.40232000 & -0.61242700 & 1.74013000 \\ 0 & -2.82094600 & -2.24878200 & -0.36447400 \\ \mathrm{H} & -3.67059800 & -2.51563700 & 0.03752600 \\ \mathrm{O} & -3.23151200 & -0.40980100 & 0.89921300 \\ \mathrm{C} & 3.07391300 & -2.02502400 & 0.62702800 \\ \mathrm{H} & 2.68248000 & -3.05177000 & 0.61801300 \\ \mathrm{H} & 3.72586900 & -1.89699900 & -0.24030900 \\ \mathrm{H} & 3.68820100 & -1.92308100 & 1.53559600 \\ \mathrm{~N} & -0.48763500 & -1.43865100 & -1.02660700 \\ \mathrm{~N} & 0.40708900 & -2.00562300 & -1.45346500\end{array}$

17

Thermal correction to Gibbs Free Energy $=0.146857$

Sum of electronic and thermal Free Energies $=-835.03026$ E_sol $=-835.3859202$ Hartree

$\begin{array}{lrrr}\text { C } & 1.33822200 & 3.04463600 & 0.26457000 \\ \mathrm{C} & 1.65627800 & 1.69589400 & 0.28463700 \\ \mathrm{C} & 0.69334600 & 0.67666600 & 0.02660600 \\ \mathrm{C} & -0.64504700 & 1.13223100 & -0.22182500 \\ \mathrm{C} & -0.93367600 & 2.51835300 & -0.27373100 \\ \mathrm{C} & 0.03446400 & 3.47538900 & -0.03279100 \\ \mathrm{C} & -1.80292700 & -1.09331400 & 0.33347600\end{array}$




$\begin{array}{lrrr}\mathrm{C} & 2.51729300 & -1.14247300 & -0.09363100 \\ \mathrm{C} & 1.10747200 & -0.71505800 & -0.12853500 \\ \mathrm{C} & -1.81091000 & 0.26319400 & -0.35680300 \\ \mathrm{O} & -2.82551700 & 0.58346300 & -1.01037000 \\ \mathrm{O} & -3.09970700 & -1.65557200 & 0.25394100 \\ \mathrm{O} & 2.75957800 & -2.40445200 & -0.56700100 \\ \mathrm{O} & 3.48906300 & -0.51222100 & 0.33924100 \\ \mathrm{C} & -1.40303300 & -1.09232300 & 1.80602300 \\ \mathrm{~N} & 0.33954700 & -1.75739100 & -0.55931300 \\ \mathrm{~N} & -0.91935400 & -1.95538500 & -0.50535500 \\ \mathrm{H} & 2.11631400 & 3.77242800 & 0.48145500 \\ \mathrm{H} & 2.67408800 & 1.40836500 & 0.49844000 \\ \mathrm{H} & -1.95788800 & 2.81774900 & -0.47214200 \\ \mathrm{H} & -0.21377600 & 4.53265600 & -0.05420800 \\ \mathrm{H} & -3.57042900 & -1.15710000 & -0.44651800 \\ \mathrm{H} & 1.87394600 & -2.74874500 & -0.85144900 \\ \mathrm{H} & -2.07761800 & -0.43257200 & 2.36091100 \\ \mathrm{H} & -1.50522600 & -2.11166300 & 2.19349900 \\ \mathrm{H} & -0.37700500 & -0.76059700 & 1.96822500\end{array}$

\section{TS5}

Thermal correction to Gibbs Free Energy $=0.144482$

Sum of electronic and thermal Free Energies $=-835.000613$ E_sol $=-835.351179$ Hartree

$\begin{array}{lrrr}\text { C } & -1.79742200 & 2.84626000 & -0.08554100 \\ \mathrm{C} & -1.88958900 & 1.45934700 & -0.05954400 \\ \mathrm{C} & -0.74117500 & 0.64159100 & -0.00913200 \\ \mathrm{C} & 0.53252300 & 1.28162000 & -0.01498300 \\ \mathrm{C} & 0.59957200 & 2.69295400 & -0.04856100 \\ \mathrm{C} & -0.54385900 & 3.47546800 & -0.07311200 \\ \mathrm{H} & -2.70721500 & 3.43963100 & -0.12514700 \\ \mathrm{H} & -2.87117800 & 1.00261300 & -0.08390000 \\ \mathrm{H} & 1.57781400 & 3.16211400 & -0.04947600 \\ \mathrm{H} & -0.46835400 & 4.55899200 & -0.09084300 \\ \mathrm{C} & 1.83825600 & -0.95606300 & 0.23518600 \\ \mathrm{C} & -2.20479300 & -1.45818300 & 0.15989500 \\ \mathrm{C} & 1.82769900 & 0.55849700 & -0.01850500 \\ \mathrm{O} & 2.89525700 & 1.15535500 & -0.22417600 \\ \mathrm{O} & 1.16135300 & -1.30064700 & 1.39772500 \\ \mathrm{O} & -2.46999000 & -2.53768200 & -0.61874200 \\ \mathrm{H} & -1.71767400 & -2.65401000 & -1.24540500 \\ \mathrm{O} & -3.04156100 & -1.11417200 & 0.99170900 \\ \mathrm{C} & 3.23744500 & -1.54496700 & 0.22886000 \\ \mathrm{H} & 3.16397500 & -2.62965600 & 0.35853900 \\ \mathrm{H} & 3.75456000 & -1.33350300 & -0.71190100 \\ \mathrm{H} & 3.81874600 & -1.12649900 & 1.05525600 \\ \mathrm{~N} & 1.13691300 & -1.55583800 & -1.01586900 \\ \mathrm{~N} & -0.10627100 & -1.55813800 & -1.03756600\end{array}$



$-0.86282600-0.84516500$
0.00552000
$\mathrm{H}$
$-0.10062300-1.15285100$
0.97429400

\section{8}

Thermal correction to Gibbs Free Energy $=0.147794$

Sum of electronic and thermal Free Energies $=-835.002996$

E_sol $=-835.3551493$ Hartree

$\begin{array}{lrrr}\text { C } & -1.98857900 & 2.68371800 & -0.24701700 \\ \mathrm{C} & -1.99455200 & 1.29500300 & -0.10821500 \\ \mathrm{C} & -0.80331900 & 0.57518500 & 0.05636600 \\ \mathrm{C} & 0.42964500 & 1.27860800 & 0.06162200 \\ \mathrm{C} & 0.41190100 & 2.68045100 & -0.07721900 \\ \mathrm{C} & -0.77937400 & 3.38324000 & -0.22719400 \\ \mathrm{H} & -2.92877600 & 3.21265300 & -0.37677800 \\ \mathrm{H} & -2.94610400 & 0.77511000 & -0.12573200 \\ \mathrm{H} & 1.35587600 & 3.21443000 & -0.07889200 \\ \mathrm{H} & -0.76404900 & 4.46372000 & -0.33813200 \\ \mathrm{C} & 1.98272300 & -0.89653500 & 0.07803800 \\ \mathrm{C} & -2.18614500 & -1.56287000 & 0.27312300 \\ \mathrm{C} & 1.78351200 & 0.64184500 & 0.14135900 \\ \mathrm{O} & 2.77735500 & 1.34854200 & 0.34151500 \\ \mathrm{O} & 1.64594300 & -1.36121300 & 1.33159300 \\ \mathrm{O} & -2.53166200 & -2.38122800 & -0.73404500 \\ \mathrm{H} & -1.77854900 & -2.41231800 & -1.37406700 \\ \mathrm{O} & -2.94771900 & -1.38156300 & 1.21073200 \\ \mathrm{C} & 3.42323000 & -1.24592000 & -0.30150400 \\ \mathrm{H} & 3.51601200 & -2.33737400 & -0.33466800 \\ \mathrm{H} & 3.69577800 & -0.84096300 & -1.28376200 \\ \mathrm{H} & 4.11364100 & -0.85652000 & 0.44962300 \\ \mathrm{~N} & 1.16217100 & -1.48379600 & -1.02583300 \\ \mathrm{~N} & -0.07255600 & -1.55038500 & -0.96419500 \\ \mathrm{C} & -0.81025500 & -0.92824800 & 0.19813500 \\ \mathrm{H} & -0.25517500 & -1.20264100 & 1.10928000\end{array}$

\section{TS6}

Thermal correction to Gibbs Free Energy $=0.146386$

Sum of electronic and thermal Free Energies $=-835.001491$ E_sol $=-835.3535883$ Hartree

$\begin{array}{lrrr}\text { C } & 3.43126400 & 0.65519000 & 0.07608400 \\ \mathrm{C} & 2.22255900 & 1.23855000 & 0.44232300 \\ \mathrm{C} & 1.00303600 & 0.53694500 & 0.36433600 \\ \mathrm{C} & 1.03530400 & -0.82066000 & -0.03935400 \\ \mathrm{C} & 2.27494800 & -1.40992100 & -0.36766100 \\ \mathrm{C} & 3.45880900 & -0.68379400 & -0.33602300 \\ \mathrm{C} & -1.46852200 & -1.15649800 & -0.46794100 \\ \mathrm{C} & -0.99746600 & 2.07924400 & -0.20335100 \\ \mathrm{C} & -0.14768100 & -1.72157500 & -0.07371800 \\ 0 & -0.02640600 & -2.93098800 & 0.23472700\end{array}$




$\begin{array}{rrrr}0 & -1.52100400 & -0.11366100 & -1.21882900 \\ 0 & -0.25493000 & 2.43244000 & -1.27224000 \\ 0 & -2.12020200 & 2.52825800 & -0.00364600 \\ \mathrm{C} & -2.64043300 & -2.11730000 & -0.48934500 \\ \mathrm{~N} & -1.22976000 & 0.50033000 & 1.72205800 \\ \mathrm{~N} & -1.64844800 & -0.58373200 & 1.41277200 \\ \mathrm{C} & -0.21145100 & 1.29010300 & 0.82623600 \\ \mathrm{H} & 4.34765400 & 1.23773300 & 0.11845500 \\ \mathrm{H} & 2.21126000 & 2.27024100 & 0.78460400 \\ \mathrm{H} & 2.28662100 & -2.45341900 & -0.66717600 \\ \mathrm{H} & 4.39604900 & -1.15495300 & -0.61944700 \\ \mathrm{H} & -0.79600800 & 3.00939300 & -1.84814300 \\ \mathrm{H} & -2.61168600 & -2.68737000 & -1.42713100 \\ \mathrm{H} & -2.62669200 & -2.82308900 & 0.34422900 \\ \mathrm{H} & -3.57209100 & -1.54264700 & -0.46555900 \\ \mathrm{H} & 0.13046900 & 2.05782700 & 1.52675800\end{array}$

19

Thermal correction to Gibbs Free Energy $=0.145345$

Sum of electronic and thermal Free Energies $=-835.015555$ E_sol $=-835.365727$ Hartree

$\begin{array}{lrrr}\text { C } & 3.37679000 & -0.45716600 & -0.30455300 \\ \mathrm{C} & 2.56826800 & 0.57928300 & 0.16193900 \\ \mathrm{C} & 1.20635200 & 0.34766500 & 0.39835600 \\ \mathrm{C} & 0.65321900 & -0.92051800 & 0.20044400 \\ \mathrm{C} & 1.47691500 & -1.96167900 & -0.24712200 \\ \mathrm{C} & 2.82757700 & -1.73038900 & -0.51109100 \\ \mathrm{C} & -1.71747500 & -0.88886000 & -0.74870100 \\ \mathrm{C} & -0.30404900 & 2.23337800 & -0.21375000 \\ \mathrm{C} & -0.81757000 & -1.15164300 & 0.49206000 \\ \mathrm{O} & -1.14072100 & -2.42622000 & 0.84431100 \\ \mathrm{O} & -1.24733600 & -0.93637300 & -1.88608200 \\ \mathrm{O} & 0.54014400 & 3.18813300 & -0.64396300 \\ \mathrm{O} & -1.40246300 & 2.03093400 & -0.70009900 \\ \mathrm{C} & -3.19994600 & -0.74073000 & -0.52089200 \\ \mathrm{~N} & -0.78959300 & 0.92580300 & 1.81098900 \\ \mathrm{~N} & -1.28530200 & -0.19196100 & 1.61528600 \\ \mathrm{C} & 0.30948100 & 1.43499400 & 0.92889800 \\ \mathrm{H} & 4.43084900 & -0.27721300 & -0.49869800 \\ \mathrm{H} & 2.98597800 & 1.56633100 & 0.34336500 \\ \mathrm{H} & 1.04839200 & -2.94850500 & -0.38991700 \\ \mathrm{H} & 3.45468100 & -2.54087900 & -0.87367700 \\ \mathrm{H} & 0.14446000 & 3.64585000 & -1.41389800 \\ \mathrm{H} & -3.75254100 & -1.15853300 & -1.36796100 \\ \mathrm{H} & -3.52202700 & -1.20956900 & 0.41068000 \\ \mathrm{H} & -3.42213700 & 0.33205400 & -0.46019000 \\ \mathrm{H} & 0.88225200 & 2.13576000 & 1.53951400\end{array}$


Thermal correction to Gibbs Free Energy $=0.142663$

Sum of electronic and thermal Free Energies $=-800.777611$

E_sol $=-801.1244635$ Hartree

\begin{tabular}{lrrr}
$\mathrm{C}$ & -3.52231900 & 0.63531100 & -0.08931300 \\
$\mathrm{C}$ & -2.32953000 & 1.35982300 & -0.06786400 \\
$\mathrm{C}$ & -1.10075700 & 0.69310000 & 0.00026900 \\
$\mathrm{C}$ & -1.07325600 & -0.71900000 & 0.05015400 \\
$\mathrm{C}$ & -2.27475100 & -1.43751500 & 0.03430100 \\
$\mathrm{C}$ & -3.49491000 & -0.76381400 & -0.03891600 \\
$\mathrm{H}$ & -4.47237000 & 1.15918800 & -0.14359000 \\
$\mathrm{H}$ & -2.34337900 & 2.44485600 & -0.09690500 \\
$\mathrm{H}$ & -2.24574800 & -2.52215200 & 0.06672300 \\
$\mathrm{H}$ & -4.42309400 & -1.32771600 & -0.05839300 \\
$\mathrm{C}$ & 1.47974000 & -0.68693300 & -0.32293000 \\
$\mathrm{C}$ & 1.44870300 & 0.69313200 & 0.40100600 \\
$\mathrm{C}$ & 0.16867200 & 1.46345000 & 0.01792100 \\
$\mathrm{C}$ & 0.22068200 & -1.45066700 & 0.12316400 \\
$\mathrm{O}$ & 0.27587800 & -2.61878600 & 0.50050800 \\
$\mathrm{O}$ & 0.19643800 & 2.68700800 & -0.15342900 \\
$\mathrm{O}$ & 1.30305700 & -0.49128300 & -1.74453900 \\
$\mathrm{H}$ & 1.93356500 & 0.20722900 & -2.00253500 \\
$\mathrm{O}$ & 2.58775100 & 1.42180600 & -0.12498700 \\
$\mathrm{H}$ & 2.34819200 & 2.36764200 & -0.09193300 \\
$\mathrm{O}$ & 1.42672900 & 0.59006200 & 1.75178500 \\
$\mathrm{C}$ & 2.75516800 & -1.46501500 & -0.03904800 \\
$\mathrm{H}$ & 3.61254400 & -0.92000900 & -0.44374600 \\
$\mathrm{H}$ & 2.89902400 & -1.60120500 & 1.03682200 \\
$\mathrm{H}$ & 2.70779000 & -2.44951400 & -0.51360800 \\
& & & \\
\hline
\end{tabular}

\section{TS1'}

Thermal correction to Gibbs Free Energy $=0.140000$

Sum of electronic and thermal Free Energies $=-800.757483$

E_sol $=-801.1031782$ Hartree

$\begin{array}{lrrr}\text { C } & 3.48683900 & 0.65411000 & 0.02316900 \\ \text { C } & 2.29066500 & 1.36709100 & 0.01642600 \\ \text { C } & 1.06620400 & 0.67991500 & -0.01007700 \\ \text { C } & 1.05126200 & -0.73254500 & 0.00032600 \\ \text { C } & 2.26097200 & -1.44032000 & -0.00877200 \\ \text { C } & 3.47174600 & -0.75007700 & 0.00778000 \\ \text { C } & -1.37934600 & -0.77745800 & 0.62472400 \\ \text { C } & -1.38783700 & 0.77845400 & -0.75182900 \\ C & -0.20511500 & 1.43503400 & -0.05095800 \\ C & -0.25868400 & -1.43067500 & -0.03005800 \\ 0 & -0.42482900 & -2.42840600 & -0.77860100 \\ 0 & -0.33820700 & 2.52181700 & 0.53000400 \\ 0 & -1.23514100 & -0.23323300 & 1.91943300 \\ 0 & -2.60524600 & 1.40552800 & -0.44978000\end{array}$




$\begin{array}{rrrr}0 & -1.29816100 & 0.33988000 & -1.94445100 \\ \mathrm{C} & -2.73798400 & -1.39387400 & 0.47904800 \\ \mathrm{H} & 4.43384100 & 1.18625100 & 0.03570700 \\ \mathrm{H} & 2.29246300 & 2.45316800 & 0.00888900 \\ \mathrm{H} & 2.24225700 & -2.52636000 & -0.00910900 \\ \mathrm{H} & 4.40801400 & -1.30122800 & 0.01390500 \\ \mathrm{H} & -0.29721000 & -0.11332200 & 2.15079300 \\ \mathrm{H} & -2.56519300 & 1.77674200 & 0.45139500 \\ \mathrm{H} & -2.77466800 & -2.37184100 & 0.97908700 \\ \mathrm{H} & -3.49235600 & -0.74546100 & 0.93361200 \\ \mathrm{H} & -2.98680800 & -1.54056200 & -0.57579200\end{array}$

11'

Thermal correction to Gibbs Free Energy $=0.140667$

Sum of electronic and thermal Free Energies $=-800.768372$

E_sol $=-801.1157367$ Hartree

$\begin{array}{lrrr}\mathrm{C} & -3.37253100 & 0.73202900 & 0.13715000 \\ \mathrm{C} & -2.15046500 & 1.38753100 & 0.26767700 \\ \mathrm{C} & -0.95592700 & 0.66358900 & 0.11125600 \\ \mathrm{C} & -0.99657200 & -0.73118500 & -0.08578400 \\ \mathrm{C} & -2.23124700 & -1.37741300 & -0.25076200 \\ \mathrm{C} & -3.41266200 & -0.64776600 & -0.13525300 \\ \mathrm{H} & -4.29950600 & 1.29160200 & 0.23056200 \\ \mathrm{H} & -2.11188500 & 2.45791900 & 0.45016900 \\ \mathrm{H} & -2.25671900 & -2.44904500 & -0.42941200 \\ \mathrm{H} & -4.37139900 & -1.14922100 & -0.23856100 \\ \mathrm{C} & 1.29182300 & -0.96082600 & 0.78218000 \\ \mathrm{C} & 1.38151400 & 1.10131700 & -0.91509000 \\ \mathrm{C} & 0.36091600 & 1.34150400 & 0.16329300 \\ \mathrm{C} & 0.30158600 & -1.43833200 & -0.11885300 \\ 0 & 0.53704000 & -2.33174400 & -1.00864100 \\ \mathrm{O} & 0.61246400 & 2.23629100 & 1.00826900 \\ \mathrm{O} & 2.57595000 & -1.39753100 & 0.49301500 \\ \mathrm{H} & 3.17614400 & -1.13398900 & 1.21162800 \\ \mathrm{O} & 0.87670900 & 0.58460300 & -2.06614300 \\ \mathrm{H} & 1.61535600 & 0.43994300 & -2.69013800 \\ \mathrm{O} & 2.55826700 & 1.45930200 & -0.82190400 \\ \mathrm{C} & 1.08758300 & -0.51196800 & 2.19666400 \\ \mathrm{H} & 1.68093200 & 0.38715200 & 2.42052000 \\ \mathrm{H} & 0.04174900 & -0.29281600 & 2.41511900 \\ \mathrm{H} & 1.41778000 & -1.30977900 & 2.87847900\end{array}$

\section{TS2'}

Thermal correction to Gibbs Free Energy $=-800.76981$ Sum of electronic and thermal Free Energies $=0.141709$ E_sol $=-801.1178482$ Hartree
$\mathrm{C}$
$-3.28569800$
$-0.91414500$
0.13235100
C
$-2.01546800-1.45304900$
0. 34000000 


$\begin{array}{rrrr}\text { C } & -0.88975500 & -0.63400100 & 0.17550900 \\ \text { C } & -1.05615800 & 0.73157800 & -0.11887400 \\ \text { C } & -2.33047600 & 1.26659100 & -0.35323200 \\ \text { C } & -3.44378100 & 0.43854500 & -0.22283200 \\ \text { C } & 1.21505700 & 0.91322700 & 0.73742000 \\ \text { C } & 1.40426800 & -1.14871600 & -0.89651700 \\ \text { C } & 0.51376400 & -1.13362900 & 0.31739600 \\ \text { C } & 0.21071500 & 1.47566800 & -0.14978200 \\ \text { O } & 0.47580300 & 2.37795900 & -0.99451800 \\ \text { O } & 0.80937200 & -1.95905700 & 1.25140500 \\ \text { O } & 2.53116200 & 1.26852900 & 0.45549700 \\ \text { O } & 0.85996800 & -0.62812300 & -2.02506400 \\ \text { O } & 2.54901000 & -1.59883800 & -0.88076100 \\ \text { C } & 1.02154000 & 0.78574900 & 2.22046400 \\ \text { H } & -4.16268600 & -1.54866600 & 0.23123000 \\ \text { H } & -1.89277700 & -2.50221900 & 0.59408700 \\ \text { H } & -2.44185700 & 2.31950400 & -0.59834100 \\ \text { H } & -4.44096400 & 0.84026400 & -0.38181200 \\ \text { H } & 2.57637500 & 1.58314500 & -0.46803100 \\ \text { H } & 1.54611400 & -0.61745400 & -2.72225300 \\ \text { H } & 1.31378300 & 1.72639000 & 2.70652000 \\ \text { H } & -0.01637600 & 0.56935500 & 2.47855900 \\ \text { H } & 1.65182200 & -0.02100000 & 2.61614300 \\ & & & \end{array}$

12'

Thermal correction to Gibbs Free Energy $=0.141884$

Sum of electronic and thermal Free Energies $=-800.782736$ E_sol $=-801.1297711$ Hartree

$\begin{array}{lrrr}\mathrm{C} & 3.10746700 & -1.11995700 & 0.35198000 \\ \mathrm{C} & 1.77631300 & -1.44822700 & 0.63074600 \\ \mathrm{C} & 0.78628900 & -0.50866500 & 0.34884800 \\ \mathrm{C} & 1.13622100 & 0.74026000 & -0.18471200 \\ \mathrm{C} & 2.46662300 & 1.07641200 & -0.46788700 \\ \mathrm{C} & 3.45353900 & 0.13113200 & -0.19451100 \\ \mathrm{C} & -1.20234100 & 0.86053000 & 0.42974500 \\ \mathrm{C} & -1.30132300 & -1.39062700 & -0.72223500 \\ \mathrm{C} & -0.73272000 & -0.67394000 & 0.54897500 \\ \mathrm{C} & -0.07624900 & 1.54253900 & -0.35715600 \\ \mathrm{O} & -0.21389100 & 2.58800100 & -0.99781300 \\ \mathrm{O} & -1.09640700 & -1.32435700 & 1.68168600 \\ 0 & -2.46338000 & 0.95814200 & -0.23298800 \\ \mathrm{O} & -0.81319800 & -0.91324500 & -1.88948900 \\ \mathrm{O} & -2.11258200 & -2.30246900 & -0.68659900 \\ \mathrm{C} & -1.27965800 & 1.50507700 & 1.81557900 \\ \mathrm{H} & 3.89187200 & -1.84433000 & 0.55618900 \\ \mathrm{H} & 1.51769200 & -2.41638200 & 1.05066000 \\ \mathrm{H} & 2.71576600 & 2.05252800 & -0.87542500 \\ \mathrm{H} & 4.49655400 & 0.35836700 & -0.39668200\end{array}$




$\begin{array}{rrrr}\mathrm{H} & -2.66991000 & 1.90251400 & -0.35460800 \\ \mathrm{H} & -1.24219200 & -1.38294600 & -2.63296400 \\ \mathrm{H} & -2.04381200 & 0.99194300 & 2.40604400 \\ \mathrm{H} & -0.32419000 & 1.44039900 & 2.34519000 \\ \mathrm{H} & -1.55109800 & 2.56213700 & 1.70697500\end{array}$

\section{TS4'}

Thermal correction to Gibbs Free Energy $=0.143973$

Sum of electronic and thermal Free Energies $=-834.991207$ E_sol $=-835.3441688$ Hartree

$\begin{array}{lrrr}\text { C } & -1.18373700 & -3.22308500 & -0.03308700 \\ \mathrm{C} & -0.02053600 & -2.47259600 & 0.12769300 \\ \mathrm{C} & -0.06434600 & -1.06818500 & 0.16962100 \\ \mathrm{C} & -1.30189900 & -0.38710200 & 0.01733900 \\ \mathrm{C} & -2.47029400 & -1.17392900 & -0.08877100 \\ \mathrm{C} & -2.41797700 & -2.56480600 & -0.12235100 \\ \mathrm{C} & -0.70745700 & 2.00592200 & -0.70165600 \\ \mathrm{C} & 2.44634000 & -0.55354700 & -0.26607900 \\ \mathrm{C} & 1.20213000 & -0.34462500 & 0.44514100 \\ \mathrm{C} & -1.43238000 & 1.07299900 & -0.01264100 \\ \mathrm{O} & -1.21311400 & 3.26940900 & -0.48142800 \\ \mathrm{O} & 3.48375800 & 0.17869500 & 0.22257700 \\ \mathrm{O} & 2.56821500 & -1.31273900 & -1.22764800 \\ \mathrm{C} & 0.43478800 & 1.85046000 & -1.64806900 \\ \mathrm{~N} & 1.21145400 & 0.56349200 & 1.39795900 \\ \mathrm{~N} & 1.18960100 & 1.36526500 & 2.20137300 \\ \mathrm{H} & -1.13134900 & -4.30801600 & -0.06110400 \\ \mathrm{H} & 0.93527700 & -2.97413700 & 0.24897300 \\ \mathrm{H} & -3.42733100 & -0.66785300 & -0.17722900 \\ \mathrm{H} & -3.33639200 & -3.13711300 & -0.22683100 \\ \mathrm{H} & 4.27255400 & -0.00235000 & -0.32510300 \\ \mathrm{H} & 1.38753700 & 2.17266400 & -1.20126800 \\ \mathrm{H} & 0.55082000 & 0.81283600 & -1.97452500 \\ \mathrm{H} & 0.26813700 & 2.47080600 & -2.53884400 \\ \mathrm{O} & -2.48226200 & 1.67691100 & 0.67467600 \\ \mathrm{H} & -2.08719700 & 2.78462100 & 0.23234100\end{array}$

16'

Thermal correction to Gibbs Free Energy $=0.142834$

Sum of electronic and thermal Free Energies $=-835.000269$ E_sol $=-835.3559908$ Hartree

$\begin{array}{rrrr}\text { C } & 1.29763500 & 3.15751300 & -0.04192200 \\ \text { C } & 0.10853300 & 2.44874200 & 0.11592500 \\ \text { C } & 0.10044100 & 1.04379000 & 0.16419300 \\ \text { C } & 1.31130200 & 0.31159900 & 0.01011700 \\ \text { C } & 2.50808100 & 1.06119600 & -0.08995000 \\ \text { C } & 2.50685400 & 2.45240900 & -0.12283600 \\ \text { C } & 0.65355100 & -2.07410800 & -0.71138400\end{array}$




$\begin{array}{rrrr}\mathrm{C} & -2.43264100 & 0.63110200 & -0.25063000 \\ \mathrm{C} & -1.19234700 & 0.37434700 & 0.45104200 \\ \mathrm{C} & 1.38837400 & -1.14909800 & -0.00906300 \\ \mathrm{O} & 0.94052500 & -3.36471800 & -0.58358900 \\ \mathrm{O} & -3.49460900 & -0.05940600 & 0.24737400 \\ \mathrm{O} & -2.53347300 & 1.39463500 & -1.21136100 \\ \mathrm{C} & -0.43489200 & -1.70720400 & -1.69295600 \\ \mathrm{~N} & -1.23086300 & -0.53200500 & 1.40469000 \\ \mathrm{~N} & -1.23179400 & -1.33428500 & 2.20804700 \\ \mathrm{H} & 1.28502800 & 4.24352800 & -0.07337300 \\ \mathrm{H} & -0.82910600 & 2.98431300 & 0.23361000 \\ \mathrm{H} & 3.44840500 & 0.52514600 & -0.17919100 \\ \mathrm{H} & 3.44676000 & 2.98941600 & -0.22503100 \\ \mathrm{H} & -4.28120000 & 0.15702200 & -0.29067600 \\ \mathrm{H} & -1.42819900 & -1.96919000 & -1.30105200 \\ \mathrm{H} & -0.44219600 & -0.64612500 & -1.95743000 \\ \mathrm{H} & -0.28748300 & -2.28756100 & -2.61313600 \\ \mathrm{O} & 2.46406900 & -1.67073800 & 0.75114000 \\ \mathrm{H} & 2.42514000 & -2.63244200 & 0.55340400\end{array}$

\section{TS5'}

Thermal correction to Gibbs Free Energy $=0.143945$

Sum of electronic and thermal Free Energies $=-835.013122$ E_sol $=-835.3668817$ Hartree

$\begin{array}{lrrr}\text { C } & 0.66628600 & 3.22039300 & 0.21474400 \\ \mathrm{C} & 1.30295000 & 1.98748100 & 0.14889100 \\ \mathrm{C} & 0.58295900 & 0.78062800 & -0.05072400 \\ \mathrm{C} & -0.83299600 & 0.88217300 & -0.17484700 \\ \mathrm{C} & -1.45574100 & 2.14765300 & -0.15713600 \\ \mathrm{C} & -0.72479300 & 3.31084400 & 0.04957100 \\ \mathrm{C} & -1.91051800 & -1.27166600 & 0.76739800 \\ \mathrm{C} & 2.65021700 & -0.73489800 & 0.01684400 \\ \mathrm{C} & 1.28323100 & -0.47593800 & -0.31614600 \\ \mathrm{C} & -1.68057100 & -0.31424400 & -0.32083500 \\ \mathrm{O} & -2.94210800 & -1.98155200 & 0.71483200 \\ \mathrm{O} & 3.17622200 & -1.88379600 & -0.54172600 \\ \mathrm{O} & 3.36642700 & -0.05950200 & 0.78105400 \\ \mathrm{C} & -0.91400400 & -1.43334400 & 1.88158800 \\ \mathrm{~N} & 0.67468400 & -1.43561200 & -1.08918100 \\ \mathrm{~N} & -0.51274000 & -1.61982100 & -1.29789300 \\ \mathrm{H} & 1.25704700 & 4.11791100 & 0.38164000 \\ \mathrm{H} & 2.38141900 & 1.94709000 & 0.23460800 \\ \mathrm{H} & -2.53384800 & 2.19828200 & -0.27445700 \\ \mathrm{H} & -1.22741800 & 4.27342800 & 0.09369100 \\ \mathrm{H} & 4.08599000 & -1.96818100 & -0.19891800 \\ \mathrm{H} & -0.01460300 & -1.94823600 & 1.52374700 \\ \mathrm{H} & -0.59449400 & -0.46207000 & 2.27282700 \\ \mathrm{H} & -1.36364300 & -2.02768700 & 2.68084300\end{array}$




$\begin{array}{llll}0 & -2.78896100 & -0.13634700 & -1.13766000 \\ \mathrm{H} & -3.41883100 & -0.84742700 & -0.89254300\end{array}$

19'

Thermal correction to Gibbs Free Energy $=0.145209$

Sum of electronic and thermal Free Energies $=-835.039495$

E_sol $=-835.3935137$ Hartree

$\begin{array}{lrrr}\mathrm{C} & 0.67688400 & 3.15222900 & 0.07596200 \\ \mathrm{C} & 1.42814200 & 1.97973700 & 0.08081400 \\ \mathrm{C} & 0.80239900 & 0.71890900 & -0.06423400 \\ \mathrm{C} & -0.60016800 & 0.68790300 & -0.22286500 \\ \mathrm{C} & -1.34440500 & 1.87436600 & -0.22492300 \\ \mathrm{C} & -0.71689700 & 3.10941300 & -0.07264500 \\ \mathrm{C} & -2.54165500 & -0.82604600 & 0.43227500 \\ \mathrm{C} & 2.91151700 & -0.75745400 & 0.09457000 \\ \mathrm{C} & 1.48133700 & -0.56617000 & -0.02084700 \\ \mathrm{C} & -1.29188000 & -0.63175100 & -0.45269600 \\ \mathrm{O} & -3.56790400 & -1.27748000 & -0.07164800 \\ \mathrm{O} & 3.33657700 & -2.06297600 & 0.17926500 \\ \mathrm{O} & 3.78247300 & 0.12550700 & 0.11566000 \\ \mathrm{C} & -2.43437700 & -0.51356300 & 1.89578700 \\ \mathrm{~N} & 0.78951200 & -1.72901800 & -0.02801300 \\ \mathrm{~N} & -0.48826500 & -1.84690100 & -0.15069100 \\ \mathrm{O} & -1.68430500 & -0.68942800 & -1.83327600 \\ \mathrm{H} & 1.18146000 & 4.10845700 & 0.19157800 \\ \mathrm{H} & 2.50255300 & 2.03071900 & 0.19977700 \\ \mathrm{H} & -2.42332100 & 1.82757800 & -0.35688000 \\ \mathrm{H} & -1.30122600 & 4.02549300 & -0.07526800 \\ \mathrm{H} & 2.51850400 & -2.61544900 & 0.14912700 \\ \mathrm{H} & -1.41421200 & -0.64270400 & 2.26664200 \\ \mathrm{H} & -2.70646800 & 0.54033100 & 2.04058200 \\ \mathrm{H} & -3.13316700 & -1.13401200 & 2.46316900 \\ \mathrm{H} & -2.49297200 & -1.23938900 & -1.87498900\end{array}$

\section{TS6'}

Thermal correction to Gibbs Free Energy $=0.139786$

Sum of electronic and thermal Free Energies $=-834.973878$ E_so1 $=-835.3209328$ Hartree

$\begin{array}{lrrr}\text { C } & 3.10746700 & -1.11995700 & 0.35198000 \\ \mathrm{C} & 1.77631300 & -1.44822700 & 0.63074600 \\ \mathrm{C} & 0.78628900 & -0.50866500 & 0.34884800 \\ \mathrm{C} & 1.13622100 & 0.74026000 & -0.18471200 \\ \mathrm{C} & 2.46662300 & 1.07641200 & -0.46788700 \\ \mathrm{C} & 3.45353900 & 0.13113200 & -0.19451100 \\ \mathrm{C} & -1.20234100 & 0.86053000 & 0.42974500 \\ \mathrm{C} & -1.30132300 & -1.39062700 & -0.72223500 \\ \mathrm{C} & -0.73272000 & -0.67394000 & 0.54897500 \\ \mathrm{C} & -0.07624900 & 1.54253900 & -0.35715600\end{array}$




$\begin{array}{rrrr}0 & -0.21389100 & 2.58800100 & -0.99781300 \\ 0 & -1.09640700 & -1.32435700 & 1.68168600 \\ 0 & -2.46338000 & 0.95814200 & -0.23298800 \\ 0 & -0.81319800 & -0.91324500 & -1.88948900 \\ 0 & -2.11258200 & -2.30246900 & -0.68659900 \\ \mathrm{C} & -1.27965800 & 1.50507700 & 1.81557900 \\ \mathrm{H} & 3.89187200 & -1.84433000 & 0.55618900 \\ \mathrm{H} & 1.51769200 & -2.41638200 & 1.05066000 \\ \mathrm{H} & 2.71576600 & 2.05252800 & -0.87542500 \\ \mathrm{H} & 4.49655400 & 0.35836700 & -0.39668200 \\ \mathrm{H} & -2.66991000 & 1.90251400 & -0.35460800 \\ \mathrm{H} & -1.24219200 & -1.38294600 & -2.63296400 \\ \mathrm{H} & -2.04381200 & 0.99194300 & 2.40604400 \\ \mathrm{H} & -0.32419000 & 1.44039900 & 2.34519000 \\ \mathrm{H} & -1.55109800 & 2.56213700 & 1.70697500\end{array}$

19"

Thermal correction to Gibbs Free Energy $=0.146399$

Sum of electronic and thermal Free Energies $=-835.041738$ E_sol $=-835.393149$ Hartree

$\begin{array}{lrrr}\text { C } & 1.00034600 & 3.18619100 & 0.24523500 \\ \mathrm{C} & 1.59400100 & 2.01166100 & -0.21260300 \\ \mathrm{C} & 0.82108400 & 0.85861100 & -0.42026500 \\ \mathrm{C} & -0.55848400 & 0.91406100 & -0.20655900 \\ \mathrm{C} & -1.16037500 & 2.09833500 & 0.24455700 \\ \mathrm{C} & -0.38147000 & 3.22876800 & 0.48242100 \\ \mathrm{C} & -1.78898600 & -1.09698200 & 0.78788500 \\ \mathrm{C} & 2.22786300 & -1.23270700 & 0.20593300 \\ \mathrm{C} & -1.38888000 & -0.30797300 & -0.49604500 \\ \mathrm{O} & -2.97579400 & -1.37612400 & 0.91855200 \\ \mathrm{O} & 2.37140900 & -2.46954000 & 0.00506200 \\ \mathrm{O} & 2.69089600 & -0.56660200 & 1.16652600 \\ \mathrm{C} & -0.74636100 & -1.50279400 & 1.77870700 \\ \mathrm{~N} & 0.51930400 & -1.32467200 & -1.60782800 \\ \mathrm{~N} & -0.70469200 & -1.31589100 & -1.41764300 \\ \mathrm{C} & 1.44744400 & -0.41262100 & -0.89939500 \\ \mathrm{O} & -2.56144400 & 0.06466100 & -1.17983300 \\ \mathrm{H} & 1.61007500 & 4.06942700 & 0.41641600 \\ \mathrm{H} & 2.66279900 & 1.97644000 & -0.40489600 \\ \mathrm{H} & -2.23365400 & 2.12518500 & 0.40581000 \\ \mathrm{H} & -0.84694100 & 4.14202900 & 0.84303300 \\ \mathrm{H} & -1.22297300 & -1.92425100 & 2.66588300 \\ \mathrm{H} & -0.09431000 & -2.26015900 & 1.32397300 \\ \mathrm{H} & -0.11210100 & -0.65355700 & 2.05052300 \\ \mathrm{H} & 2.22521800 & -0.18723600 & -1.63808000 \\ \mathrm{H} & -3.28803600 & -0.49039500 & -0.82606000\end{array}$

